\title{
Über die Histogenese und Struktur der Knorpelgrundsubstanz.
}

\author{
Von \\ K. von Korff. \\ Hierzu Tafel XI und 7 Textfiguren.
}

\section{Einleitung.}

Die erste erkennbar zwischen den heranwachsenden Knorpelzellen liegende Substanz, aus der später die Knorpelgrundsubstanz hervorgeht, wird als prochondrale Substanz (Hasse, 1879) oder auch als Vorknorpel (Studnička, 1903) bezeichnet; ihrer Struktur und Herkunft nach wird sie sehr verschieden beurteilt. Die meisten Autoren sehen die prochondrale Substanz als strukturlose Viasse an, die durch Umwandlung oder Sekretion des Protoplasmas der sich bildenden Knorpelzellen, welche ich als Vorknorpelzellen bezeichne, entsteht und eine mit den Zellen zusammenhängende (syncytiale) Masse bildet.

Die Frage nach der Bildung der Grundsubstanzfibrillen, ob primär (intracellulär) von den Bindegewebszellen oder sekundär (extracellulär) aus einer homogenen Intercellularsubstanz wird meist von den Autoren nicht erörtert oder nur vermutungsweise gestreift. Über das Verhalten der definitiven Knorpelzellen zur Intercellular- oder Knorpelgrundsubstanz, über das Wesen der sogenannten Knorpelkapseln, über ihre Zugehörigkeit zur Zelle oder Intercellularsubstanz sind die Angaben der Autoren verschieden.

Nach C. Hasse (1879) stellt die zwischen den Zellen des Blastems gelegene erste Anlage, die "prochondrale Grundsubstanz", ein Naschen- oder Alveolenwerk dar und geht aus einer „Umwandlung von Zellprotoplasma" hervor. Die wirkliche Knorpelgrundsubstanz tritt aber erst später ,in Form von Höfen (oder Kapseln) um die Knorpelzellen herum auf".

Strasser (1879) bearbeitete die Genese des byalinen hnorpels der Salamanderlarven, er fasst die erste Intercellularsubstanz als eine einheitliche, durch Umwandlung von Protoplasma 
entstandene Substanz auf: "Zwischen den Kernen kommt nur eine verbältnismässig geringe Menge hellen Protoplasmas", das "wenig optisch differenziert erscheint," vor. „Erst später treten in diesem nicht weiter differenzierten Protoplasma zwischen den einzelnen Kernen die Zellgrenzen auf, indem hier die ersten Grundsubstanzen auftreten."

Josef Schaffer (1901) untersuchte genauer den "Zellenknorpel" in den Flossenstrahlen von Petromyzon. Seine gewonnenen Anschauungen fasst $J$ osef Schaffer im wesentlichen dahin zusammen:

1. "Die erste Anlage der morphologisch als Knorpel sich abgrenzenden Zellmassen ist eine syncytiale."

2. "Die in diesem Syncytium deutlich werdenden Zellgrenzen stellen ein Fach- oder Wabenwerk dar, dessen Lücken von den kernhaltigen Zellkörpern ausgefüllt werden. Dasselbe geht teilweise aus einer unmittelbaren Umwandlung (Verdichtung) des Protoplasmas hervor; verhalt sich färberisch zunächst wie dieses und nimmt auch ferner an den Wachstumserscheinungen und Stoffwechselvorgängen desselben teil. Dieses Fachwerk, welches bereits eine dem Wachstumsdrucke entsprechende funktionelle Anordnung zeigt, bildet die prochondrale Grund- oder Kittsubstanz."

Studnička (1903) untersuchte Teleostier (Lophius) und Selachier (Torpedo, Pristiurus, Spinax). Es entsteht nach Studnička (Lophius) durch Zusammenwachsen der Zellen mit ihren Ausläufern, die kürzer und dicker werden, und schliesslich auch mit ihrem Zelleib ineinander übergehen, ein Syncytium. Sobald dies geschieht, treten scheidewände auf. „In diesem Moment erscheinen Scheidewände und die Individualităt der Zellen ist gerettet." Wenn ich Studniěka recht verstehe, so wird auch die sich zu Scheidewänden verdichtende Masse der ersten Intercellularsubstanz als Exoplasma aufgefasst.

Verschieden von den histogenetischen Differenzierungen müssen die Vorgănge im Innern des schon entwickelten Knorpels aufgefasst werden, die zu einem inneren oder interstitiellen Wachstum führen. Ich meine die Vermehrung der eben differenzierten Knorpelzellen, die von den fertigen Knorpelzellen bewirkte Sekretion, die Bildung der sogenannten Knorpelkapseln, die Ver- 
mehrung der einmal angelegten Grundsubstanzfibrillen durch sich selbst (Teilung). Diese für die inneren Wachstumsvorgänge wichtigen Fragen kommen naturgemüss für uns weniger in Betracht. Wir suchen hier das Entstehen der ersten Anlage, den morphologischen und histologischen Charakter derselben, die Difierenzierung der Grundsubstanzfibrillen, der Knorpelzellen aus dem Bindegewebe zu erkennen.

Vielfach wurde den inneren Wachstumserscheinungen histogenetische Bedeutung beigelegt, was zu einer grossen Verwirrung und $\mathrm{zu}$ falschen Auffassungen führen musste.

Äbnliche Unklarheiten existieren iiber das Verhalten der Grundsubstanzzellen, die vielfach noch beute als Grundsubstanzbildner, „Chondroblasten“; angesehen werden, zur Cirundsubstanz.

Die alte Schwannsche Ansicht ist noch heute für viele Autoren massgebend. Nach Schwann (1839) wird die Zwischensubstanz dadurch hervorgebracht, dass die Wände der Zellen sich verdicken und verschmelzen oder, was viel häufiger ist, dadurch, dass sich die Intercellularsubstanz in grosser Qualität entwickelt und eine Verschmelzung der unverdickten oder wenig verdickten Zellenwände mit der Intercellularsubstanz eintritt."

Histogenetische Bedeutung haben nur die im lockeren Bindegewebe vor sich gehenden Differenzierungsprozesse des Bindegewebes, welches noch nach vielen Richtungen hin differenzierungsfähig ist. Hier finden wir die ersten Anfünge der Histogenese. Hier können wir von Stufe zu Stufe die dicht nebeneinander liegenden Entwicklungsstadien der Grundsubstanz verfolgen. Nicht aber können wir, wie es vielfach geschehen ist, in einem mehr oder weniger fertigen Knorpelgewebe mit Betrachtungen der Knorpelzellen, die Chondrogenese verfolgen.

Die Knorpelbildung im Perichondrium, die ich hauptsächlich untersuchte, können wir mit Koelliker als indirekte Chondrogenese bezeiclunen gegenüber einer direkten oder primären Entwicklungsart. Ko elliker fasst seine Ansicht über die beiden Entwicklungsarten folgendermassen: „Bei der direkten Entstehung wandeln sich embryonale Zellenmassen dadurch in Knorpelgewebe um, dass die Zellen sich vergrössern und deutliche Membranen erhalten. Entwickeln sich die Zellen in dieser Art weiter, so entsteht der Zellenknorpel; in den meisten Fällen jedoch tritt zwischen denselben eine Zwischensubstanz auf; die in entfernter 
Linie von der alle Gewebe durchtränkenden Ernährungsflüssigkeit herzuleiten ist, aber unzweifelhaft auch unter einer gewissen Mitwirkung der Knorpelzellen sich bildet..." „Bei der indirekten Bildung des Knorpelgewebes ist der Ausgangspunkt ein bereits fertiges Gewebe und zwar entweder eine Art Bindegewebe mit kleinen Zellen oder ein Faserknorpel. Beide dieser Gewebsformen können, wie ich zuerst bei Fischwirbeln nachwies, in echten hyalinen Knorpel sich umwandeln, was später auch $\mathrm{Hasse}$ bestätigte. Ganz Ähnliches findet sich an den Stellen, an denen Perichondrium in Knorpelgewebe sich umbildet."

\section{Hyalinknorpel.}

1. Bildung der prochondralen fibrillären Grundsubstanz im lockeren Bindegewebe.

Sehr geeignet für histogenetische Unter'suchungen des Hyalinknorpels ist der Salamanderknorpel seiner Grosszelligkeit wegen. Ich habe in erster Linie sich Hach aushreitende Knorpel von Salamanderlarven (Sternum, scapula und den Kiemenknorpel) untersucht. Die instruktivsten Bilder geben Hach oder schrilg zur Obertläche geführte Schnitte der in Zenkerscher Flüssigkeit, Sublimat, Sublimat-Alkohol-Eisessig (v. Le n hos sék) fixierten Knorpelstücke. Gefärbt babe ich vor allem mit der M. Heidenhainschen Eisenalaunbämatoxylinmethode allein oder mit nachfolgender Färbung mit Azokarmin, den Chromotropen, und der von Mallory angegebenen Bindegewebsfärbung.

Die obertlächlichen Schichten des Perichondriums der genannten hyalinen Kinorpel vom Salamander bestehen aus lockerem Bindegewebe, sie enthalten zahlreiche junge gut färbbare, meist langgestreckte Bindegewebszellen und zwischen denselben sehr viele Bindegewebstibrillen.

Die Zellen vermehren sich durch mitotische T'eilung. Zellleib wie Kern imponieren durch ihre Grösse; die Kerne haben in der Aufsicht platte, der Knorpeloberflache parallel gestreckte Flächen, in der Kantenansicht sind sie länglich oval (Fig. 1, 2 der Taf. XI). Nach der Knorpeloberfläche zu liegen die Zellen dicht gedrängt übereinander. Der Zelleib ist später wenig färbbar, mit der M. Heidenhainschen Eisenalaunhämatoxylinmethode dargestellt erscheint er blassgrau, seine Konturen treten wenig deutlich hervor. Im Protoplasma entwickeln sich jetzt sehr zahlreiche 
Körner, die mit der genannten Yethode deutlich hervortreten, auch mit der C. B end a schen Kristallviolettmethode dunkelviolett in dem sonst rötlichbraun gefärbten Plasma erscheinen. An vielen Stellen legen sich die Körner zu kettenförmigen Längsreihen dicht aneinander und durchsetzen in verschiedenen Richtungen, meist in der Längenausdehnung, den Zelleib. Wie es scheint, wachsen sie zu Plasmafibrillen zusammen. Denn in vielen Zellen sieht man nur wenig Plasmakörner, desto mehr Plasmafibrillen, die man vielfach durch mebrere mit ihren Fortsätzen anastomosierende Bindegewebszellen verfolgen kann. Das deutliche Hervortreten dieser Gebilde, ihre spezifische Fürbung spricht dafür, dass hier spezifische Bestandteile des Zelleibes, die B endaschen Mitochondrien, zur Bildung von Plasmafibrillen verwandt werden.

Die intracellulär gelegenen Fibrillen verhalten sich färberisch basophil, später, wenn sie ausserhalb des Zelleibes liegen und selbstindige Elemente geworden sind, werden sie acidophil. Sie liegen jetzt als ein für die Entwicklung der Knorpelgrundsubstanz wesentlicher Bestandteil des Perichondriums in den Lücken zwischen ihren Mutterzellen (Fig. 2 der Taf. XI) oder auch ibren Mutterzellen an.

Ihrem chemischen Verhalten nach fasse ich sie als präcollagene Bindegewebsfibrillen auf, die mit dem Stoffiwechsel ihren chemischen Charakter andern und sich durch Teilung vermehren.

Auf Fig. 2 der Taf. XI (Querschnitt durch die knorpelige Schadelkapsel der Salamanderlarve) sind sie fachwerkartig in den Lücken zwischen den Bindegewebszellen angeordnet als besondere fibrilläre Intercellularsubstanz. Sie bilden hier die erste und zwar fibrilläre Anlage der Knorpelgrundsubstanz. Dieselbe besteht aus sich durchflechtenden Fibrillenzügen, die in den tieferen Schichten des Perichondriums sich bedeutend vermehren.

In den Lücken des prochondralen fibrillären Fachwerkes liegen die Bindegewebszellen, die, wie wir gleich sehen werden, sich zu Knorpelzellen allmählich differenzieren.

Unter dem Einfluss der sich vergrössernden Vorknorpel und Knorpelzellen erweitern sich die Maschenräume, in denen sie liegen. An der Peripherie sind sie länglich und schmal, im Vorknorpel schon breiter und geräumiger, im Knorpel noch breiter mehr abgerundet. Bei dieser allmählich sich machenden Ausdehnung und Abrundung der Knorpelzellen und Maschenräume 
finden deutlich in Erscheinung tretende Verschiebungen der acidophilen Bindegewebsfibrillen statt, aus der ursprünglich langgestreckten Lage werdeı sie in bogenförmig oder auch ringförmig die Knorpelzellen umkreisende Züge verlagert. Die den Knorpelhöhlen näher gelegenen zeigen jetzt häufig das Bild einer nestartigen Durchtlechtung. Die in der Mitte zwischen den Knorpelzellen liegenden behalten mehr die Längsstreckung.

Sehr interessant ist die Anordnung der jungen Bindegewebsfibrillen der pronchondralen Substanz in den oberflachlichen Schichten des Perichondriums platter Knorpel (Fig. 3, 4 der Taf. XI). Die acidophilen Bindegewebstibrillen legen sich zu platten, der Oberfläche zunächst mehr oder weniger parallel verlaufenden Lamellen zusammen. Die Lamellen liegen in der Peripherie dichter übereinander: als in der Tiefe, sind verschieden dick, und scheinbar leicht wellig gebogen. Höchstwahrscheinlich werden die Fibrillen in den Iamellen durch eine zunächst noch wenig dichte homogene Masse, in der sie eingebettet sind, wenn auch nur locker, zusammengehalten.

Innerhalb der fibrillären Lamellen findet eine Überkreuzung der Fibrillen und Fibrillenbündel, die wahrscheinlich sich hier noch bedeutend vermehren, in verschiedenen Richtungen statt. Von den gröberen Lamellen zweigen sich feinere Seitenlamellen ab, die die Hauptlamellen verbinden oder auch geflechtartig durchsetzen. So entsteht ein Fachwerk sich durchflechtender Bindegewebslamellen. In dem Fachwerk liegen die Bindegewebszellen. Je mehr die Zellen sich zu Knorpelzellen vergrössern, findet eine Erweiterung der Fächer durch eine Dehnung oder ein Auseinanderweichen der Lamellen an den betreffenden Stellen statt.

Bei Haifischen zeigen die platten Knorpel ähnliche Anordnungen der Bindegewebsfibrillen im Perichondrium. Fig. 5 der Taf. XI bezieht sich auf den Flachschnitt des knorpligen Schädeldaches eines ca. $15 \mathrm{~cm}$ langen Acanthias vulgaris. Man sieht die zahlreichen übereinander liegenden, mit der Mallory-Methode blau gefärbten Lamellen, die obersten Schichten zeigen eine deutlichere Blaufärbung der sie zusammensetzenden Bindegewebsfibrillen, als die tiefer liegenden, welche kontinuierlich in die Grundsubstanz des Knorpels übergehen. An den schräg zur Oberfläche getroffenen Stellen zeigen die Lamellen wellenartige Erhebungen und Tiefen. Sich abzweigende Seitenlamellen und die Haupt- 
lamellen durchsetzende Nebenlamellen kommen auch hier wie beim Salamander vor. Die in den Lücken zwischen den Lamellen liegenden Bindegewebszellen verhalten sich ebenso wie beim Salamander. Es sind plasmaarme Zellen, die sich ganz allmählich $z u$ Knorpelzellen entwickeln.

Die Lamellenbildung ist, glaube ich, eine vorübergehende Erscheinung, van der Stricht (1887) beschreibt sie auch im fertigen Knorpel. Die lamellenartige Anordnung verschwindet scheints beim Übergang der Lamellen in die Knorpelgrundsubstanz, wo die Zellen zwischen denselben grösser werden.

Studnička (1903) teilt über die Entstehung der Grundsubstanzfibrillen des Selachierknorpels, iber die ich an meinen Präparaten nichts Bestimmtes aussagen kann, Näheres mit. Die Mesenchymzellen von Torpedo ocellata (12 mm lang) und Spinax niger (ca. $4 \mathrm{~cm}$ lang) differenzieren in ihrem Zelleib und Protoplasmafortsaitzen Fasern, die sich mit Säurefuchsin und anderen sauren Farbstoften intensiv färben, sie gehen von einer Zelle in die andere iiber und lassen sich auf diese Weise (nicht so die einzelnen Fibrillen wie eher die ganzen Bündel derselben) im Gewebe auf weite Strecken verfolgen, sie sind die ersten Andeutungen der collagenen Bindegewebsfasern. „Die jungen Bindegewebsfasern verlieren sich auf der Oberfläche des Knorpels in der Grundsubstanz, die ebenfalls eine doch etwas feinere Faserung aufweist." Die Zelleiber der Vesenchymzellen und deren anastomosierende Fortsätze Hiessen dann nach Studničkas Vorstellung zu einer Art von Syncytium zusammen, das die erste Anlage vorstellt. Die im jungen Bindegewebe befindlichen feinen Faserungen werden in dem erwähnten Syncytium eingeschlossen und liegen, da sie sich unterdessen noch vermehrt haben, sebr dicht aneinander und bedingen die eigentliche Struktur der Grundsubstanz des jungen Knorpels (Fig. 5 und 6, Studnička, 1903). In die Grundsubstanz des Knorpels übergehende Bindegewebsfibrillen hat auch Jos ef $\mathrm{Sch}$ a f f e r an vielen Stellen beobachtet. An den Knorpelflossenstrablen von Petromyzon fluviatilis beschreibt Schaffer (1901) dies Verbalten: „Hier kann man den Schnittrand der Knorpelsubstanz sich fortsetzen sehen in blasse Bündelchen, welche hier leicht als leimgebende Fasern des Perichondriums erkannt werden. Zwischen ihnen, sie verbindend, sieht man mit Orcein dunkel gefärbte Streifen, welche manchmal leicht körnig erscheinen. 
Weiter in den Knorpel hinein verliert sich diese Unterscheidbarkeit der zwei Längsstreifen, die Grundsubstanz nimmt das bekannte homogene Aussehen an." Josef Schaffer gibt über dies Verhalten die Deutung, „dass von Zellen des Perichondriums zwischen die umgebenden Fibrillenzüge hinein eine mit Orcein, Hämalaun etc. färbbare Kittsubstanz abgeschieden wird, welche schliesslich die collagenen Bündel so durchtrånkt, dass sie unsichtbar werden und mit der Kittsubstanz eine bomogene Masse bilden; es ist dies ein Assimilationsvorgang . .." An anderer Stelle spricht Schaffer von einer chondrogenen Metamorphose der Bindegewebsfibrillen. Auf Seite 136 (l. c.) werden die zwischen den Vorknorpelzellen liegenden Fibrillenzüge als "fremdartige Scheidewände" aufgefasst, die nach Schaffers Auffassung durch die assimilatorische Finigkeit der jungen Knorpelzelle der „chondrogenen Metamorphose" zugeführt werden.

Wir müssen vermuten, dass mit den Assimilationsprozessen $\mathrm{Sch}$ a f f ers Autlösungsprozesse gemeint sind, wodurch die Fibrillen zu einer einheitlichen homogenen Masse zusammenfliessen, um zur Grundsubstanz. zu werden.

Wir wissen aber, dass die Knorpelgrundsubstanz fibrillär ist. Um das fibrillare Stadium zu erlangen, würde sich also nach der Schafferschen Ansicht eine fibrilläre Masse in eine homogene und dann wieder in eine fibrilläre umwandeln müssen. $\mathrm{Zu}$ dieser sonderbaren Annahme liegt jedoch kein Grund vor.

Bei Besprechung des aus verschiedenen Bindegewebsarten hervorgehenden postembryonalen Knorpels von Cyclostomen ist Studnička (1897) bezüglich der Bedeutung der zwischen den Vorknorpel- bezw. Knorpelzellen beobachteten fibrillären Intercellularsubstanz in den einzelnen Fallen verschiedener Ansicht.

Im „festen fibrösen Bindegewebe “ (den Fascien, Studnička 1. c.) geraten die Bindegewebsfasern am Anfang der Knorpelbildung weiter voneinander , und werden endlich zwischen den dicht aneinander anliegenden Knorpelzellen ganz aufgelöst, an ihrer Stelle entwickelt sich zwischen den Zellen, aber erst später, die Grundsubstanz des Knorpels".

Auf Seite $627 \mathrm{ff}$. wird dagegen von Studnička der direkte Zusammenhang von Bindegewebsfasersträngen des lockeren Binde gewebe mit den Fasern der Knorpelgrundsubstanz konstatiert und als histogenetisch aufgefasst. „Es ist vielleicht nicht nötig, hier 
besonders $\mathrm{zu}$ bemerken, dass es sich da um eine Bildung des Knorpels aus dem Bindegewebe und nicht um einen umgekehrten Prozess handelt."

Sehr bald geht das von mir beschriebene erste tibrilläre Stadium der prochondralen Substanz in ein zweites, homogen aussehendes über. Diese Veränderung geht mit einem Wechsel der mikrochemischen Reaktion vor sich, d. h. die Acidophilie verschwindet, es tritt eine Basophilie auf. Bevor ich auf diese mit der Bildung der Kittsubstanz und der Chondroitinschwefelsïure (C. $\mathrm{H}$ ans e $\mathrm{n}, 1905$ ) zusammenhängende chemische Veränderung der Grundsubstanz eingehe, wollen wir das Schicksal der Bindegewebszellen, die sich zu Knorpelzellen differenzieren, ins Auge fassen.

\section{Die Differenzierung der Bindegewebszellen zu}

Knorpelzellen, Wachstum des Knorpels.

Wie wir erwähnten, verlieren die Bindegewebszellen während der Bildung der Bindegewebsfibrillen ibren Zelleib ganz oder bis auf einen kleinen Rest. So liegen sie als "nackte Kerne" (St udnička, 1903) oder plasmaarme Zellen zwischen ihrem Produkt; ten Bindegewebsfibrillen des Perichondriums (Fig. 2, 3 der Taf. XI). Sehr bald findet jedoch eine Regeneration ilres Zelleibes statt, wobei wohl nur die plasmaarmen Zellen in Betracht kommen. Dieser Vorgang, der zur Bildung von Knorpelzellen in den Lücken der sich durchflechtenden Fibrillenzüge führt, lüsst sich in den Einzelheiten schwer verfolgen. Bei Salamanderlarven geht die Differenzierung in den Hauptzügen folgendermassen vor sich. Der länglich ovale grosse Kern verkleinert sich bedeutend; früher sehr dünn, mit platten breiten Flïchen, wird el jetzt mehr und mehr abgerundet. Ob hierbei ein Teil durch Abschnürung verloren geht, oder nur eine Verdichtung des Chromatin- und Liningerüstes unter gleichzeitiger Umlagerung und Verschiebung der Kernsubstanzen vor sich geht, lässt sich nicht mit Bestimmtheit sagen. Viele Kerne zeigen Einbuchtungen. Nach erfolgtel Abrundung und Verkleinerung des Kernes wird ein heller, schmaler. wenig färbbarer Hof als primäre Masse des Zelleibes der zukünftigen Knorpelzelle sichtbar. In dieser Plasmasubstanz, die wahrscheinlich aus dem Plasmarest der Fibroblasten hervorgegangen ist, entwickeln sich mehr und mehr sehr feine Körner, die sich mit der 
Mall o ry-Methode rot, mit Eisenalaunhämatoxylin schwärzlich färben. Bei weiterer Differenzierung erfolgt eine Vergrösserung des Zelleibes, eine Vermehrung der Plasmakörner und schliesslich erscheinen Plasmafasern (Filarsubstanz), welche meist radiär angeordnet sind (Textfig. 1).

B ig e to w (1879) beschreibt die Veranderungen der Knorpelzellen in der Knorpelanlage der Scapula von Triton folgendermassen: „In den mittleren Schichten haben die Zellen wie die Kerne im

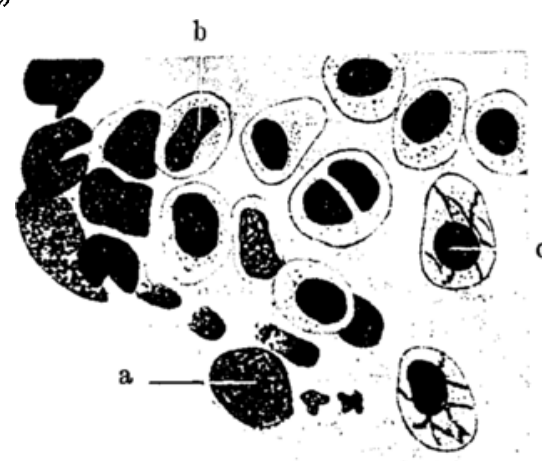

Fig. 1.

Bildung der Krorpelzellen aus Bindegewebszellen. $\mathrm{a}=$ plasmaarme Bindegewebszellen; $\mathrm{b}=$ Vorknorpelzelle; $\mathbf{c}=$ Knorpelzelle der Salamanderlarve

(Kiemenknorpel). allgemeinen eine rundliche Form und sind reich an Protoplasma, weiter gegen die Oberflache hin erscheinen sie mehr und mehr abgeplattet

c mit ihren breiten Flachen der Obertliche parallel gelagert; die Kerne werden im Verhältıis zum Zellprotoplasma immer grösser. so dass hier zahlreiche Zellen vorhanden sind, bei denen das Protoplasma kaum zu erkennen ist und die Kerne fast den ganzen Zelleib einnehmen. Dicht unter dem Perichondrium wird die Form derZellen eine ganz unregelmässige."

Die Regeneration des Zelleibes tritt jedoch nur bei wenigen Zellen ein; die meisten Bindegewebszellen und die Kerne derselben zerfallen nach der Fibrillenbildung und werden, wie es scheint, resorbiert. Man findet verschiedene Anzeichen des Verfalles, besonders an den Kernen, die "nackt" erscheinen, Verlust der Färbbarkeit des Chromatins, Auflösung des Chromatinnetzwerkes, tiefe Einschnürungen, die zu Abtrennungen von Kernsubstanz führen. Die Regelmässigkeit der Form, das längliche Oval des Kernes verliert sich vor dem Zerfall, neben stark ein geschnürten Kernen finden sich solche mit langen dünnen lappigen Fortsätzen.

Der Auffassung Studničkas (1911), dass "nackte Kerne" (Kerne ohne Plasma) des Bindegewebes unter direkter Neubildung 
des Zelleibes zu Knorpelzellen heranwachsen, kann ich nicht ohne weiteres beistimmen. Den Prozess beschreibt der Autor im einzelnen: „An der Oberfläche des Zellkernes erscheint anf einmal eine feine aussere Membran, welche sich von ihm immer mehr abhebt, so dass zwischen beiden ein im Leben wohl mit einer Flüssigkeit ausgefüllter Raum zustande kommt. In diesem sieht man, nachdem der Zellbildungsprozess etwas weiter fortgeschritten ist, spärliches Protoplasma. Es ist an der Kernoberfläche angehäuft und verbindet in der Gestalt von feinen, kaum sichtbaren Strängen den Kern mit der frïher erwähnten Membran, welche nichts anderes ist, als die primäre Kapsel der neuen Knorpelzelle..." „.Dass die auf einmal massenhaft erscheinende und jene Kapsel spannende ZellHlüssigkeit vom Kern produziert wird, ist so wie so sicher, und von dem Zellplasma der Knorpelzelle muss man schliesslich dasselbe annehmen. " Ich habe unlängst für diese Tätigkeit des Zellkernes den Namen „cytoblastische Funktion" angewendet und ich bleibe dabei.

Studnička zitiert die Beobachtungen Goettes über diese Art von Zellregeneration, aus denen hervorgeht, "dass die Emibryonalzellen dem späteren Knorpelgewebe nur die Zellkerne unmittelbar überliefern, nicht aber zugleich die zugehörigen Zellenleiber".

Zellteilungen tinden in dem fertigen Knorpel nicht mehr statt, dagegen trifft man die Vorknorpelzellen oder die eben differenzierten Knorpelzellen nicht selten in mitotischer Teilung. Nach der Teilung liegen die Tochterzellen in einer gemeinsamen Höhle meist zu zweien, bei weiterer Teilung der Tochterzellen zu Vierer- oder Achtergruppen sich gegenüber. Das von diesell Zellgruppen besetzte Gebiet ist das Zellterritorium der Autoren.

Nach den Untersuchungen von Clapariède (185T). R. He id en ha in $(1861,1863)$ und $\mathrm{Sch}$ le i cher (1879) werden die Knorpelhöhlen durch eigenartige Zelldifterenzierung der sich teilenden Knorpelzellen, aus welcher Scheidewandbildungen hervorgehen, in zwei, vier, acht usw. Fächer geteilt. Es ist nämlich eine Eigentümlichkeit des Knorpels, dass die Teilung des Zelleibes seiner Zellen "nicht durch Einschnürung geschieht, sondern dass zuvor eine Scheidewand sich bildet, die sich später in zwei Blitter spaltet" (Schleicher 1. c.). Bei dem von mir untersuchten Salamander- wie Selachier-Hyalinknorpel, dem elastischen Netz- 
knorpel des Ohres von jungen Kaninchen, kommen fast regelmässig nur einmalige Teilungen der jungen Knorpelzelle in einer gemeinsamen Knorpelhöhle (Grundsubstanzlücke) vor. Die Knorpelhöhle ist meist länglich oval, die sich bildende Scheidewand steht dann senkrecht zur Längsachse.

Die Einzelheiten bei der Scheidewandbildung innerhalb der Knorpelhöhle entnehme ich den Mitteilungen Schleichers, der an lebenden Knorpelzellen diesen sehr interessanten und eigenartigen Prozess schrittweise sich abspielen sah: „Die erste Anlage zur Bildung der künftigen Scheidewand sehen wir durch eine längliche Reihe von feinen, seitlich aneinander gelegenen Fädchen dargestellt. Ausnahmsweise erscheint sie zuweilen sehr spat, wenn die beiden Kerne schon am Ende ihrer Teilung stehen; meist tritt sie jedoch kurz nach der Teilung auf, in einer Ebene, die in der Mitte zwischen den sich bildenden neuen Kernen liegt. Dass die Elemente zur Scheidewandbildung dem Plasma entlehnt werden, lassen uns Beobachtungen voraussetzen, in welchen wir amöboiden, im Protoplasma gelegenen Fiidchen eine Richtung zur

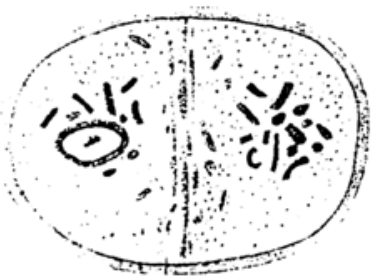

$\mathrm{g}^{\prime}$
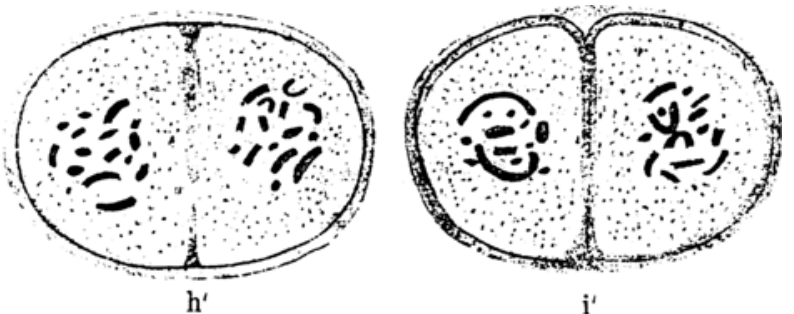

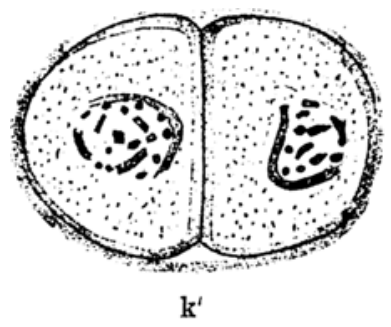

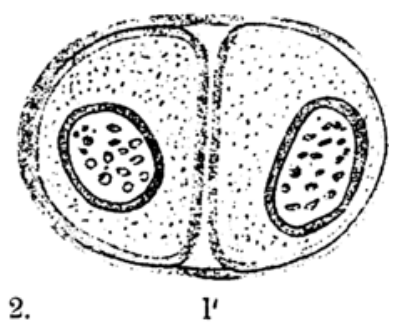

Mittelebene des Zellkörpers zuerkennen müssen. Auch sahen wir solche Fadchen den schon entstandenen sich anschmiegen. Es entstand auf diese Weise eine doppelt konturierte Membran, in welcher eine einfache Linie erschien, welche sich in zwei gleich 
dicke parallele Blätter teilte. Die Trennung dieser Blatter beginnt mit dem Auseinanderweichen an ihren beiden Enden, und an diesen divergierenden Enden sieht man nun die beiden Blatter sich mit einer inzwischen formierten Tochterkapsel in Verbindung setzen."

Die nebenstehend wiedergegebenen Figuren $\mathrm{g}^{\prime}-\mathrm{l}^{\prime}$ der Schleicherschen Arbeit geben die Bildung der Knorpelhöhlenscheidewand während der Endstadien der mitotischen Teilung (Telophase) wieder.

Hiernach will es allerdings scheinen, als ob nach der Spaltung der Scheidewand die beiden Teilungshalften derselben ganz in die Kapselsubstanz der Tochterzellen aufgenommen werden und dann keine Scheidewand in der Knorpelhöhle der Mutterzelle mehr existiert, sondern nur eine einheitliche Knorpelhöhle.

Nach diesen Beobachtungen Schleichers sind die sogenannten Knorpelkapseln als Differenzierungsprodukt des Zellleibes und zum Teil als Produkt der Zellteilung der jungen Knorpelzellen aufzufassen.

Sollen wir nun die Vorknorpel oder jungen linorpelzellen und deren Teilungen in Beziehung zur Grundsubstanzbildung bringen? Fraglos führen sie zur Vermehrung der Zellen, zur Bildung der Knorpelscheidewände, bei mehrfacher Teilung zur Bildung der Knorpelzellennester, der Zellterritorien, welche vielfach der Grundsubstanz das charakteristische Aussehen geben. Über die sogenannten Knorpelzellenkapseln, ihre Natur, ihre Herkunft habe ich trotz vieler Beobachtungen nichts ausmachen könnell. Ich muss annehmen, dass in vielen Fallen, wo die Autoren die Kapsel beschreiben, nur ein chemisch differenter Teil der Grundsubstanz vorhanden ist. Viele Autoren nehmen an, dass die Knorpelzellen periodisch eine geschichtete Kapselsubstanz ausscheiden, die nach und nach zur Grundsubstanz wird, sie geben sogar in der Theorie so weit, dass sie den Knorpelzellen - sie werden direkt als Chondroblasten (Schaffer l. c.) bezeichnet - die alleinige Bildung der Knorpelgrundsubstanz zuschreiben. Die Autoren vermuten, dass die Knorpelzellen eine homogene Intercellularsubstanz bilden, die später in die Grundsubstanzfibrillen zerfällt. Ich bezeichne diese Theorie der Autoren als "Chondroblastentheorie". Diese Theorie entbehrt jeglicher Begründung, sie ist mit den von mir gemachten Beobachtungen unvereinbar, vor allem sieht man nichts, was als Sekret der Knorpelzellen aufgefasst werden muss. 
Koelliker unterscheidet (Gewebelehre, 6. Auti., I.Bd., S. 107) an den Knorpelzellen zwei Teile, die eigentliche Zelle wird Protoblast (Knorpelkörperchen der Autoren) genannt und ist ein membranloses Gebilde mit hellem Protoplasma, zu dieser Zelle gehört als zweiter Teil „die äussere Zellmembran oder die Knorpelkapsel, eine durch Ausscheidung des Protoblasten gebildete feste belle oder gelbliche Lage, welche diesen dicht umgibt und durch fortgesetzte Ausscheidung der Protoblasten, die an ihrer inneren Oberflache sich ansetzen, ein geschichtetes Ansehen und eine sehr bedeutende Dicke erlangen kann. Derartiges habe ich an den Knorpelzellen nicht erkennen können.

Dass die definitiven Knorpelzellen keine Grundsubstanz im histogenetischen Sinne bilden können und überhaupt nicht mehr teilungsfahig sind, geht aus den Beobachtungen Peyrands (Comptes r'cndus, t. 84, p. 1308, 1577) hervor; nach denen zwar eine Regeneration wabren Knorpels vorkommt, aber nicht von den alten Knorpelzellen, sondern stets vom Perichondrium aus erfolgt.

Mit den Erfahrungen Pey rands stimmen die genauen und interessanten Untersuchungen $\mathrm{Sch}$ walbes über Knorpelregeneration (1878) überein. $\mathrm{Zu}$ diesem $Z$ wecke wurden in Kaninchenohren mit dem Locheisen runde Löcher geschlagen. Die Löcher wurden durch Zuwachs von dem Wundrand aus verengt und schliesslich ganz geschlossen. Das junge Gewebe bestand aus jungem Knorpelgewebe. Die mikroskopische Untersuchung ergab, dass sich das neugebildete Knorpelgewebe scharf gegen den alten Knorpel absetzt und an der Grenze keine Zellteilungen des alten Kuorpels vorkommen. Dagegen fand Schwalbe, dass das Perichondrium des alten Knorpels sich kontinuierlich in das junge Knorpelgewebe fortsetzt; seine zelligen Elemente gehen ganz allmählich unter Umwandlung ihrer Form in Knorpelzellen, seine Grundsubstanz unter Aufhellung in Knorpelgrundsubstanz über.

Mit diesen Ergebnissen stimmen die exakten Beschreibungen F. Marchands (1901) über die bei der Heilung der Knorpelwunden beobachteten Knorpel-Neubildungsprozesse, wie wir später sehen wer'den, überein.

Über den Modus des Knorpelwachstums gaben folgende Versuche Schwalbes Aufschluss. Es wurden in Kaninchenohren mehrere Löcher mit dem Locheisen geschlagen und deren Ent- 
fernung gemessen, gleich nach der Operation und viele Wochen später. Hierbei ergab sich, dass der Abstand der Löcher, von Mitte zu Mitte gemessen, stets dieselbe Entfernung beibehält, während die Ohrmuschel selbst an Umfang zunimmt. Hiermit war der Beweis erbracht, dass das Flächenwachstum des Ohrknorpels jedenfalls nicht interstitiell erfolgt, sondern durch Apposition an den Randpartien.

Schwalbe räumt indessen ein, dass er nicht gewillt sei, die am elastischen Ohrknorpel gewonnenen Resultate ohne weiteres auf alle Knorpel zu übertragen, dass vielmehr das Wachstum der embryonalen Skelettknorpel ein anderes sei und hier neben einem appositionellen ein interstitielles Wachstum vorkommt, das sich sowohl in der Zunahme der Intercellularsubstanz, als in der Vermehrung der in dieselbe eingebetteten Kinorpelzellen ausspricht.

Nach meinen histogenetischen Untersuchungen findet jedoch auch im embryonalen Knorpel, sobald derselbe ein bestimmtes Alter und völlig entwickelte "grossblasig" aussehende Knorpelzellen hat, kein nennenswertes interstitielles Wachstum mehr statt, sondern auch hier bedingt die Apposition das Wachstum der Grundsubstanz so gut wie ganz allein.

Man kann hier nun einwenden, die Zellnester, die doch aus den Teilungen der Knorpelzellen hervorgegangen sind, finden sich nicht nur in den äusseren perichondralen Appositionsschichten, sondern auch mehr mitten im Knorpel selbst. Trotzdem haben die Zellteilungen der zentral gelegenen Zellnester in den Randpartien des Knorpels stattgefunden, nur durch die Verbreiterung des Knorpels durch Appositionsschichten sind die Zellnester mehr und mehr zu zentral liegenden geworden. Die so bewirkte, mehr gleichmässige Verteilung der Zellnester der Grundsubstanz ist deshalb nicht als Beweis dafür aufzufassen, dass die Zellteilung auch bej alten Knorpelzellen statt hat.

3. Das sekundäre, homogen aussebende Stadium der Chondrogenese, die Kittsubstanz der Autoren, die Basophilie der Knorpelgrundsubstanz.

Die histochemisch und farbanalytisch sehr wertvollen Arbeiten von Mörner (1888, 1889), Hammar (1894), Hansen (1905) ergaben, dass die ausgesprochene Basophilie (Fürbbarkeit mit 
Hämalaun, Hämatoxylin) der definitiven Grundsubstanz an die Kittsubstanz gebunden ist. Entfernt man mit $10 \%$ Kochsalzlösung, mit Kalk- oder Barytwasser die Kittsubstanz (H a m mar, 1. c.), so verschwindet die Tingibilität für Hämatoxylin. Die Untersuchungen von $\mathrm{Hansen}$ (l. c.) ergaben, dass hauptsächlich in der Kittsubstanz, ein Specificum des Knorpelgewebes, die Chondroitinschwefelsäure lokalisiert ist und die Basophilie der Knorpelgrundsubstanz bedingt. Han se n äussert sich darüber folgendermassen : „Da die Affinitït des Knorpels zu basischen Farbstoffen durchweg bedeutend grösser war als die des Kernchromatins, dessen Baso. philie wohl von der Nucleinsaure herrührt, und da der Gedanke a priori eine gewisse Wahrscheinlichkeit hat, dass eine so entschiedene Affinitat eines Gewebes zu exquisit basischen Farbstoffen von dem Vorhandensein einer Säure im Gewebe, die Fürbung mithin wahrscheinlich von einer Art Salzbildung herriihrt, und da ferner Mörner und Schmiedeberg ja eben im Knorpel das Vorhandensein reichlicher Chondroitinschwefelsäure nachgewiesen haben, und da keiner der anderen chemischen Hauptbestandteile des Knorpels, weder Albumin (Albumoid), noch Kollagen, besonders starke basophile Eigenschaften besitzt, so lag mir der Schluss nahe: Die Basophilie der Knorpelgrundsubstanz ist wahrscheinlich dem Vorhandensein der Chondroitinschwefelsäure zu verdanken."

Zum Beweise wurden von Hansen (l. c.) frischen oder fixierten Knorpelschnitten in schonender Weise, so dass die Intertibrillarsubstanz erhalten blieb, durch Zusatz von Reagentien $1 \%$ - $3 \%$ Kali oder Natronlauge oder durch jahrelanges Liegenlassen in ca. $45 \%$ Alkohol die Chondroitinschwefelsiture entzogen. Die so behandelten Schnitte hatten ihre Basophilie velloren; sie wurden dann mit einer Lösung von Chondroitinschwefelsäure imbibiert und die Basophilie trat wieder auf (Hansen, l. c., S. $587 \mathrm{ff}$.).

Hansen glaubt, dass die Chondroitinschwefelsiure in erster Linie an die Eiweißstoffe oder Albumine der "Kittsubstanz" gebunden sei.

Gegenüber den beschriebenen Fibrillen ist die Kittsubstanz ein sekundares Produkt: sie maskiert die Fibrillen, so dass die Grundsubstanz schon sehr bald homogen erseheint. Ich möchte glanben, dass dies sowie das Nichtanwenden von zuverlässigen Bindegewebsfärbungen die Autoren über die wahre Natur der Knorpelanlage getäuscht hat. 
Wie ich erwähnte, ist das erste fibrilläre Stadium der Chondrogenese acidophil, was offenbar auf die präcollagenen oder collagenen Bindegewebsfibrillen, die im wesentlichen die prochondrale Substanz zusammensetzen, zurückzuführen ist. Sobald eine Verdichtung des fibrillären Geflechtes durch Ablagerung der Kittsubstanz der Autoren (Homogenisierung der Grundsubstanz) eintritt, verliert sich die Acidophilie, das Gewebe zeigt immer grössere Affinitüt zu basischen Farbstoffen. Dies ist das sekundäre Stadium der Chondrogenese (Fig. 6 der Taf. XI).

Über die Bildung der Kittsubstanz sagen meine Präparate nichts aus. Doch liegt die Vermutung nahe, dass sie ein Produkt der Vorknorpel- und linorpelzellen ist. Die Maskierung der acidophilen Bindegewebsfibrillen geht nämlich immer erst an denjenigen Stellen vor sich, wo die Vorknorpelzellen in die Knorpelzellen übergehen. Ferner ist in der Nähe der Knorpelzellen die Basophilie und Maskierung der Fibrillen meist stärker als in den entfernter liegenden 'Teilen der Intercellularsubstanz.

Bei línochenfischen finden sich dieselben Verhältnisse der Histogenese wie bei Haifischen und Amphibien. Textfig. 3 illustriert die allmählich vor sich gehende Differenzierung des Bindegewebes in Knorpelgrundsubstanz in den Hauptzügen bei einem ca. $3 \mathrm{~cm}$ langen Forellenembryo (Hyalinknorpel der Schädelkapsel, Fixierung v. Le n hos sék sches Sublimat-Alkohol-Eisessiggemisch, MalloryFärbung).

Zwischen den plasmaarmen Bindegewebszellen des Perichondriums liegt eine acidophile fibrilläre Intercellularsubstanz, die nach der Peripherie mit den Bindegewebsfibrillen des umgebenden Bindegewebes zusammenhängt. Sie bildet ein Fachwerk von sich durchtfechtenden Fibrillenzügen als erste Grundsubstanzanlage. Nach dem Knorpel hin geht das Fachwerk in die Grundsubstanz des Knorpels ïber. Währenddessen vergrössern sich die Bindegewebszellen in den Lücken des Fachwerkes. Die Lücken werden zu Knorpelböblen, die Zellen unter starker Vergrösserung z.u Knorpelzellen. Die fibrillare Struktur der prochondralen Grundsubstanz verschwindet bei der Umwandlung zur Knorpelsubstanz. Es tritt eine Maskierung der Fibrillen durch die Kittsubstanz ein, die Grundsubstanz erscheint homogen.

Ich möchte hier kurz anf die Schaffersche Ansicht von dem Knorpelbildungsprozess bei Petromyzon eingehen. Die Zellen Archiv f. mikr. Anat. Bd.84. Abt. I. 
der Anlage der knorpeligen Flossenstrahlen von Ammocoetes liegen nach Schaffer (1901) so dicht aufeinander gepresst, dass "von Zellgrenzen keine Spur zu sehen ist und die Kerne sich oft mit ihren Membranen unmittelbar berühren". Die Zellen nehmen an Grösse $z u$, „erscheinen teils wie aufgequollen, so dass der Kern derselben rings von einem deutlichen, wenn auch schwach färbbaren Protoplasmakörper umgeben wird und werden durch scharfe Grenzen voneinander getrennt". „Diese Grenzen werden

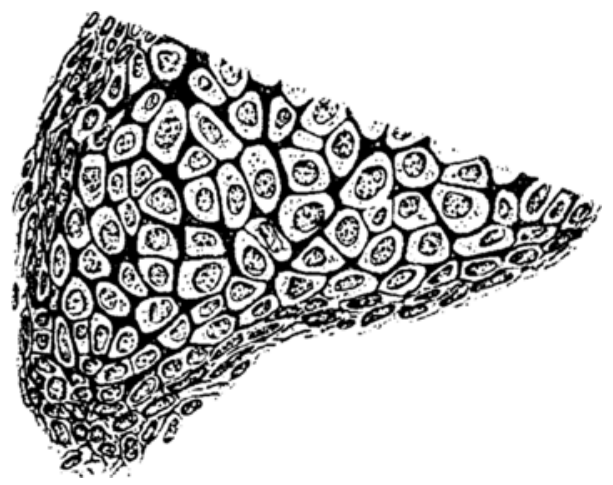

Fig. 3.

durch eine verdichtete und stärker licbtbrechende Rindenzone des Protoplasmas, die sich mit Eosin stärker rot färbt, gebildet und sind stets zwei benachbarten Zellen gemeinsam, so dass sie wie ein Fachwerk oder Alveolensystem die Zellkörper umschliessen." Schaffer sieht diese balkenartig angeordnete Substanz als Intercellularsubstanz an, die von den "Zellkörpern gebildet wird und die Zellkörper wie ein lebendiger Kitt verbindet" und zur prochondralen Substanz wird.

Wenn wir genauer diese Beschreibung ins Auge fassen, so ergibt sich, dass nach Schaffer Intercellularsubstanz dasselbe ist wie Syncytium! Intercellularsubstanz müsste sich aber gegen die Zellen abgrenzen. Wie soll aus einem Syncytium eine Intercellularsubstanz werden? Wir können uns deshalb nichts Bestimmtes unter der von Schaffer geschilderten Intercellularsubstanz, die auch als "Kittsubstanz" bezeichnet wird, vorstellen.

Allerdings müssen wir zugeben, dass die Knorpelanlage der Schwanztlossenstrahlen von Ammocoetes histologisch schwer zu deuten ist. Meiner Meinung nach gehört überhaupt dies blasige 
Gewebe nicht zum Knorpelgewebe; jedenfalls dürfen wir es nicht mit dem Hyalinknorpel in eine Gruppe stellen. Auch Studnička hat sich, wenn ich recht erinnere, in seinen Arbeiten über die Histogenese der Cyclostomenknorpel gegen diese Auffassung Schaffers ausgesprochen.

Meine Petromyzon-Präparate betreffen den Schwanzflossenknorpel von 5-12 cm langen Ammocoetes Planeri (Fixierung Zenkersche Flüssigkeit und Sublimat). Bei den verschiedensten Färbungen (Delafield sches Hämatoxylin, M a ll oryfärbung, Eisenalaunhamatoxylin ohne oder mit Bindegewebsfärbung, Resorcinmethode nach Weigert) ist es mir nicht gelungen, eine besondere Intercellularsubstanz, die man etwa als erste Grundsubstanzanlage auffassen kann, fürberisch darzustellen, wenigstens nicht auf den Stadien, wo Schaffer die erste Anlage beschreibt. $\mathrm{W}^{\top}$ as hier von den blasigen Zellen differenziert wird, ist weiter nichts als eine membranartige Aussenschicht. Dieselbe färbt sich intensir mit Resorcin, älunlich der Elastica interna der Chorda dorsalis. Ein Übergang derselben in eine Intercellularsubstanz findet nicht statt. Ob dieses eigenartige Gewebe als sogenanntes vesikulöses Stützgewebe aufzufassen ist, vermag ich einstweilen nicht zu sagen. In seiner Struktur zeigt es infolge der Zusammensetzung aus blasig aussehenden Zellen viel Ähnlichkeit mit dem Chordagewebe, das ja auch verkehrterweise von vielen Autoren als Knorpelgewebe angesehen wird.

\section{Elastischer Netzknorpel.}

1. Embryonale Anlage.

Bei der noch wenig entwickelten Knorpelanlage der Ohrmuschel von Kaninchenembryonen (ca. $10 \mathrm{~cm}$ lang) und Schweinsembryonen (ca. $12 \mathrm{~cm}$ lang) zeigt sich folgendes: Das noch sehr junge Bindegewebe hat sich in der Mitte zu einer wellig gebogenen muschelförmigen Bindegewebsplatte verdichtet, der Anlage des elastischen Knorpels. In derselben liegen die sehr kleinen Bindegewebszellen dicht gedrängt. Ihr Zelleib ist stark reduziert, wahrscheinlich nach vorhergegangener Fibrillenbildung; die Kerne sind abgerundet, die Zellen trifft man vielfach in mitotischer Teilung. In dem in Textfig. 4 wiedergegebenen Präparate wurde die Mallorysche Bindegewebsfärbung angewandt. Sie zeigt deutlich, dass zwischen den kleinen plasmaarmen Vorknorpelzellen schon 
eine differenzierte Intercellularsubstanz gebildet ist. Es ist dies eine deutlich fibrilläre bindegewebige Substanz, mit geschlängelt verlaufenden Bindegewebszügen, auch einzeln verlaufenden Bindegewebsfibrillen, die Fibrillen färben sich nur schwach mit Anilinblau (Mallory); mit der Resorcin-Säurefuchsinfärbung nach We ig e r t tritt auch nur eine geringe Farbreaktion auf. Hiernach will es scheinen, dass die Intercellularsubstanz der Anlage des elastischen Netzknorpels aus Fibrillen besteht, die noch keinen bestimmten chemischen Charakter" haben.

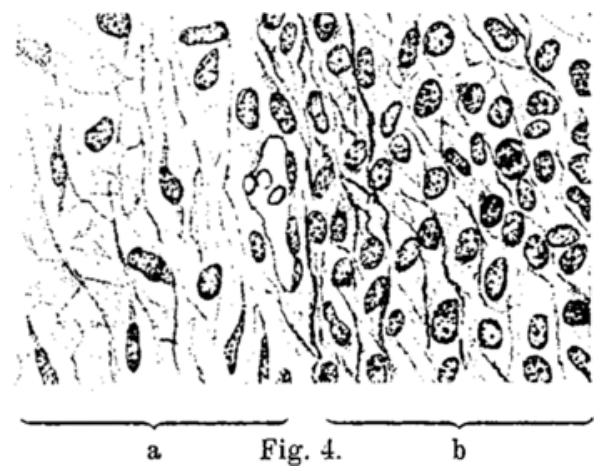

In der Umgebung der Knorpelanlage beginnt die Differenzierung des Bindegewebes zum Perichondrium =a. Die in ihm liegenden Bindegewebszellen sind plasmareich, meist spindelförmig, die zwischen den perichondralen Bindegewebszellen liegenden Fibrillen und Fibrillenzüge färben sich stärker mit Anilinblau als die des umgebenden Mesenchymgewebes; an vielen Stellen gehen sie über in das fibrilläre Fachwerk der ersten Knorpelanlage $=b$. Eine anders geartete Intercellularsubstanz, die man als erste Grundsubstanzanlage auffassen könnte, lässt sich an den zahlreichen Präparaten, die ich anfertigte, nicht nachweisen. Färbungen mit den von $\mathrm{M}$. $\mathrm{He}$ ide $\mathrm{nh}$ ain eingeführten Chromotropen, Azokarmin, Benzo-Lichtbordeaux, lassen ebenfalls den fibrillären Charakter der Intercellularsubstanz erkennen (Textfig. 4).

Für die von vielen Autoren, z. B. Heinrich Müller, Reichert, Koelliker, Frey, Leydig (die betreffende Literaturangabe findet sich in der Arbeit O. Hertwigs, 1873), vertretene Anschauung, dass sich die elastischen Fasern aus einer homogenen Intercellularsubstanz durch eine Art Härtung 
und Verdichtung bilden sollen, findet in meinen Untersuchungen keinen Anhaltspunkt, weder bei embryonalem noch postembryonalem Ohrknorpelgewebe.

o. Hertwig (1873) schliesst aus seinen Beobachtungen an sich entwickelndem Ohrknorpelgewebe von neugeborenen Kaninchen und menschlichen Embryonen, dass es sich bei der Bildung der elastischen Fasern nicht um die Umwandlung einer vorher gebildeten homogenen Knorpelgrundsubstanz handelt, sondern um eine formative Tätigkeit des Protoplasmas. Seiner Ansicht nach entstehen die elastischen Fasern an der Obertlache der Zellen in dichter Anlagerung an das Zellprotoplasma, sie laufen über die quergestellten Reihen der Knorpelzellen weg. Ich möchte glauben, dass an den Stellen, wo O. Hertwig die elastischen Fasern zuerst sah, dieselben nicht mehr primär sind, sondern bereits verdickt in der Intercellularsubstanz liegen; wenigstens sind die noch ganz jungen, im postembryonalen Perichondrium gelegenen elastischen Fasern meiner Präparate eines 6 Wochen alten Kaninchens sehr dünn und lassen sich im ungefürbten Zustande nicht erkennen, was bei den älter'en und verdickten Fasern jedoch bei ihrem starken Lichtbrechungsvermögen (in Wasser oder Glycerin) leicht möglich ist.

Prinzipiell dagegen stimme ich mit den O. Hertwigschen Ergebnissen, dass die elastische Faser ein Produkt des Protoplasmas der Zellen ist, überein. Jedoch wird sie nach meinem Dafürhalten nicht in ihrer Eigenschaft als elastische Faser von den Knorpelzellen gebildet, sondern als indifferente Bindegewebsfibrille von den gewöhnlichen Bindegewebszellen und wandelt sich erst später in die elastische Faser um.

\section{Die postembryonale Bildung.}

Bei älterem postembryonalem Ohrknorpel des Kaninchens (ca, 6 Wochen alt) untersuchte ich in Zenkerscher Flüssigkeit und Sublimat fixierte Präparate. Als beste Färbung erwies sich Vorfärbung mit Boraxkarmin 24 Stunden, Nachfärbung mit Resorcin - Săurefuchsin nach Weigert. Die Präparate zeigen, wie schon Schwalbe (1. c.) und Sieveking (1892) experimentell nachwiesen, dass das Knorpelwachstum hier ein rein appositionelles ist, indem das Bindegewebe des Perichondriums unter Umwandlung seiner Zellen in Knorpelzellen kontinuierlich in die Grundsubstanz des Knorpels übergeht. 
Im Perichondrium liegen in der aussersten Schicht zahlreiche collagene Bindegewebsbündel, in der inneren Schicht fast nur elastische Fibrillen. Mit der Resorcin-Säurefuchsinmethode, besonders nach längerem Liegenlassen der gefärbten Schnitte in Wasser, treten die collagenen Fasern wenig in Erscheinung, um so deutlicher die elastischen Fasern.

Die Bindegewebsfasern des Perichondriums sind mit denen der Knorpelgrundsubstanz kontinuierlich. Bei diesem Übergang geht dem färberischen Verhalten nach eine mikrochemische Veränderung, ferner eine Vermehrung und ein Dickenwachstum der Bindegewebsfasern vor sich. In den äusseren Schichten des Perichondriums sind sie nur schwach färbbar mit Resorcin, sehr dünn und spärlich, nach dem Knorpelrande zu in der Gegend des Vorknorpels sind sie zahlreicher, dicker, ihr netzartiges Gefüge oder Zusammenwachsen wird deutlicher; sie bilden hier eine besondere Intercellularsubstanz, durchsetzen die Lücken zwischen den sich entwickeInden Knorpelzellen fachwerkartig. Nach der Differenzierung der Knorpelzellen aus den Bindegewebs- resp. Vorknorpelzellen bilden die elastischen Fasern die geformten Bestandteile der Netzknorpelgrundsubstanz. Zur Zeit der definitiven Verhältnisse entwickelt sich zwischen den Knorpelzellen eine anders furbbare homogene Intercellularsubstanz, in welche die elastischen Fasern eingelassen sind. Ich fasse diese homogene Masse als Analogon der Kittsubstanz des Hyalinknorpels auf, welche jedoch die elastischen Fasern nur umgibt, nicht, wie es scheint, durchtrünkt. Ein "Maskieren" der elastischen Fasern durch diese Kittsubstanz findet nicht statt. Dies Verhalten gibt Kopsch in "R a uberKopsch", Lehrbuch der Anatomie, wieder (9. Aufl., I. Abt., Fig. 120).

Dass diese Kittsubstanz erst nach der Differenzierung der Knorpelzellen und zwar in ihrer unmittelbaren Nâhe sichtbar wird, spricht für die Annahme, dass die Knorpelzellen diese färbbare homogene Substanz bilden. Die Zellen des Ohrknorpels nehmen in der postembryonalen Wachstumsperiode bedeutend an Umfang zu, nach 0 . Hertwig (l. c.) bis zum fünffachen ibres ursprünglichen Volumens. Zellteilungen finden nur in den jungen, am Rande liegenden Knorpelzellen statt, nicht aber bei den alten, völlig entwickelten Knorpelzellen.

Das elastische Fasernetz wird in späteren Differenzierungsstadien der Knorpelgrundsubstanz durch eine homogene, kapsel- 
artige Substanz, die an Breite zunimmt, immer weiter von der Zelle entfernt. Nach 0 . Hertwigs Untersuchungen besteht diese breite ringförmige Masse aus abwechselnd helleren und dunkleren Streifen und ist ein Produkt des Protoplasmas. Auch nach meinen Untersuchungen gehört diese kapselartige Masse nicht zur Intercellularsubstanz, sondern ist als eine Art Exoplasma der Knorpelzelle aufzufassen, das in der sich erweiternden Knorpelhöble liegt, was auch, wie mir scheint, die 0 . Hertwigschen Abbildungen, Fig. 7, 14, zeigen.

\section{Faser- oder Bindegewebsknorpel.}

Die Bindegewebsknorpelbildung habe ich im Discus intervertebralis ron ca. $30 \mathrm{~cm}$ langen Kalbs- und ca. $17 \mathrm{~cm}$ langen Schweinsfeten nüher untersucht. Die noch sehr weichen Zwischenwirbelscheiben wurden in Zenkerscher oder Flemmingscher Flüssigkeit fixiert, die Schnitte wurden mit Eisenalaunhämatoxylin vor- und mit Chromotrop nachgefärbt. Nach Fixierung mit Zenkerscher Flüssigkeit wurde auch die Mallorysche Färbemethode angewandt.

In der Umgebung des als Nucleus pulposus persistierenden mittleren Abschnittes der Zwischenwirbelscheibe liegen noch indifferente, meist sternförmige, weniger zahlreiche spindelförmige Bindegewebszellen. In ihrem Zelleib differenzieren sich zahlreiche Bindegewebsfibrillen. Dieselben verlieren sehr bald ibren $\mathrm{Zu}-$ sammenhang mit dem Protoplasma, sie liegen dann als besondere Masse zwischen den Bindegewebszellen und bilden ein Fachwerk sich filzartig durchflechtender Fibrillenbündel. In den Fächern der fibrillären Intercellularsubstanz wachsen die Bindegewebszellen ganz allmählich zu Knorpelzellen heran. Hierbei findet eine Abrundung des Protoplasmas statt, welches an Volumen zunimmt und sich mit einer kapselartigen Substanz umgibt. Die zwischen ihnen liegende fibrilläre Intercellularsubstanz wird zur Grundsubstanz des Bindegewebs- oder Faserknorpels unter Beibehaltung ihres fibrillären Charakters. Eine färbbare Kittsubstanz oder homogene Grundsubstanz wird also beim Faserknorpel nicht gebildet. Daher ist es im allgemeinen viel leichter, die Chondrogenese des Faserknorpels zu verfolgen, als die des Hyalinknorpels. Den Bindegewebsknorpel können wir nach den obigen Betrachtungen als eine Vorstufe des hyalinen Knorpels auffassen; Hyalinknorpel geht 
aus Faserknorpel dort hervor, wo sich die knorplig präformierten Wirbelkörper der Wirbelsäule plazieren. Schon K o elliker (l. c.) erwähnt, dass beim Fischwirbel der Faserknorpel sich in echten Hyalinknorpel umwandelt. Dieselben Übergänge kann man sehr deutlich auf Längsschıitten durch sich entwickelnde Hirschgeweihstangen (Baststangen) erkennen, bei der Entwicklung der Substantia spongiosa, der die Bildung eines Hyalinknorpels vorausgeht.

Die zirkulär annähernd parallel zur ausseren Obertlïche verlaufenden Lamellen des Discus intervertebralis, die durch schräg verlaufende Faserzüge sich verbinden, entstehen bauptsïchlich durch Apposition von innen nach aussen unter gleichzeitig vor sich gehendem interstitiellem Waclistum; die einzelnen Appositionslamellen werden weiter und breiter, was nur durch eine selbständige Vermehrung und das Längen- und Dickenwachstum der Fiblillen zu erklïren ist. Eine Differenzierung oder Prigung der Grundsubstanzfibrillen aus einer homogenen Intercellularsubstanz, wie die Auhänger der extracelluläı'en Genese der Bindegewebstibrille annelımen, ist hier ausgeschlossen. Denn es kommt hier überhaupt nicht zur Bildung einer besonderen farbbaren Intercellularsubstanz.

Der als Nucleus pulposus bezeichnete Teil des discus intervertebralis ist nicht als Rest der Chorda dorsalis - dieselbe verschwindet schon sehr früh im Zentrum der Zwischenwirbelscheibe - aufzufassen, wie es die Lehrbücher tun, sondern als Rest der skelettogenen Schicht der Chorda, welcher nach vollendetem Wachstum der Zwischenwirbelscheibe sich nicht weiter zum Faserknorpel differenziert, sondern seinen primären Charakter, den des lockeren Bindegewebes, bewahrt. Während des Wachstums der Zwischenwirbelscheibe differenziert sie immer neue Appositionslamellen, den annulus tibrusus, von innen nach aussen.

An vielen anderen Stellen kann man den kontinuierlichen Übergang von Bindegewebsfibrillen einer präformierten Bindegewebsar't in die Grundsubstanzfibrillen des Kuorpels konstatieren. So bei der Achillessehne der Frösche. Die Fibrillen und Fibrillenzüge derselben laufen hier durch die Lücken zwischen den Knorpelzellen des „Sesa mschen Knorpels" und werden so zu Grundsubstanzfibrillen des Knorpels; andere Grundsubstanzfibrillen existieren nicht. Die Knorpelzellen sind auch hier histogenetisch modifizierte Bindegewebszellen. die einen grossen blasig aussehenden Zelleib entwickeln. Soviel ich an den einzelnen mit Eisenalaunbämatoxylin 
Über die Histogenese und Struktur der Knorpelgrundsubstanz. 287

gefärbten, in Sublimat fixierten Schnitten sehen kann, tragen die grossen Zellen nicht den typischen Knorpelzellencharakter, d. h. sie differenzieren keine als Kinorpelkapsel zu bezeichnende Aussenschicht, sondern nur eine sehr dünne, scheinbar elastische Membran, liegen nicht in Knorpelhöhlen. Die Fibrillen der Grundsubstanz laufen in starken, meist weit getrennten Bündeln und werden nicht durch Bildung einer homogenen Kittsubstanz maskiert.

Dieses eigenartige Gewebe hat immer Schwierigkeiten bezüglich der Klassifizierung gemacht. Stade Ima nn (1878) bezeichnet den „Sesamschen Knorpel“ als Pseudoknorpel, Renaut (1893) als "Tissu fibrobyalin".

Studnička reiht diesen Knorpel ein in eine besondere Gruppe, die des "Vorknorpelgewebes". Zur Prizisierung des "Vorknorpelgewebes" führt Studnička (1903) an, dass es „einmal nur schnell vorübergehend bei der Chondrogenese auftritt, ein anderes Mal wieder etwas länger bleibt, in vielen Fällen sogar lebenslang erhalten bleibt. Durch das Auftreten von Bindegewebsfisern zwischen den Zellen, die zu einer Festigung des Gewebes dienen, kirin das Gewebe im letzteren Falle mehr oder weniger modifiziert werden, ohne deshalb die charakteristischen Eigenschaften zu verlieren. Immer bemerken wir, dass ein bleibendes Vorknorpelgewebe aus denselben Geweben seinen Ursprung nehmen kaun, aus denen unter anderen Umständen sich ein Kinorpel entwickelt, und auch im bleibenden Vorknorpelgewebe können wir in sehr vielen Füllen noch immer die Tendenz der einzelnen Zellen, sich in Knorpelzellen umzuwandeln, bemerken."

Ein weiteres Beispiel des Überganges und der Umwandlung von faserigem Bindegewebe in die Grundsubstanz des hyalinen linorpels ist der Gelenkknorpel. Dieses Verhalten demonstriert ein Schnitt durch den Gelenkknorpel eines neugeborenen Hundes (Fig. 7 der Taf. XI). Am Rande des hyalinen Knorpels haben sich die Bindegewebsfibrillen des Perichondriums lamellenartig der Oberfläche parallel gelagert. Die so verlaufenden Hauptzüge geben seitlich sich abzweigende Fibrillenbündel ab oder werden von anderen Fibrillenzügen in verschiedenen Richtungen fachwerkartig durchsetzt. So entsteht ein Trabelwerk. In dem Fachwerk der sich durchflechtenden Fibrillenzüge und Lamellen liegen nach der Peripherie noch spindelförmige Bindegewebszellen; nach dem Knorpel zu runden sie sich ab und werden zu Knorpelzellen. Die 
Räume (Fächer) zwischen den sich kreuzenden Fibrillenzügen werden grösser, sie werden zu Knorpelhöhlen, welche von den heranwachsenden Knorpeizellen ausgefüllt werden. Gleichzeitig mit der Entwicklung der Knorpelzellen findet eine Vermehrung der beschriebenen Fibrillenzüge, die wir als fibrilläre prochondrale Intercellularsubstanz des Gelenkknorpels ansehen müssen, statt. In die eigentliche Knorpelgrundsubstanz lassen sich nun in diesem Falle die Fibrillen der prochondralen Intercellularsubstanz auf weite Strecken hin verfolgen und liegen hier in einer andersgearteten Masse, der homogenen Kittsubstanz.

\section{Zusammenfassung.}

Die erste Anlage der Knorpelgrundsubstanz, der' "Vorknorpel ${ }^{*}$ oder die "prochondrale Substanz", ist nicht homogen, sondern setzt sich aus acidophilen Bindegewebsfibrillen zusammen, die von indifferenten Bindegewebszellen gebildet werden. Nach der Fibrillenbildung wandeln sich die Fibroblasten in Vorknorpel- und Knorpelzellen um. Zwischen den Vorknorpelzellen bilden die Bindegewebsfibrillen ein Gerüstwerk sich durchHlechtender acidophiler Fibrillenzüge, das prochondrale intercelluläre Gerüstwerk des Knorpels, das unter Vermehrung der Fibrillen an Masse zunimmt. Die Maschenräume oder die Fächer der sich durchflechtenden Fibrillenzïge sind die primären Knorpelhöhlen, die sich beim Grösserwerden der Vorknorpel- resp. Knorpelzellen erweitern. Gleichzeitig mit der Erweiterung der Knorpelhöblen findet eine Verschiebung oder Umlagerung der Fibrillen des Gerüstwerkes der prochondralen Grundsubstanz statt. Dies ist das erste fibrilläre Stadium der Knorpelanlage.

Durch die Ablagerung einer homogenen Kittsubstanz von Seiten der Knorpelzellen werden die Grundsubstanzfibrillen des Hyalinknorpels "maskiert"; die Grundsubstanz erscheint homogen, wird basophil. Dies ist das zweite basophile Stadium des Hyalinknorpels. Beim Faserknorpel und elastischen Netzknorpel findet keine Maskierung der Grundsubstanzfasern durch die Interfibrillarsubstanz statt; sie bleiben histogenetisch auf dem ersten fibrillären Stadium des Hyalinknorpels stehen.

Die typischen Knorpelzellen finden sich in dem ersten Stadium der Histogenese überhaupt nicht, sondern erst im letzten, ihre Funktion ist nicht erkennbar, doch liefern sie wahrscheinlich die 
Kittsubstanz oder auch die Chondroitinschwefelsäure. Zellteilungen finden sich nur bei jungen eben differenzierten Knorpelzellen, bei perichondraler Chondrogenese trifft man sie nur am Rande des Knorpelgewebes. Aus diesen Teilungen gehen die Zellterritorien der Knorpelgrundsubstanz hervor, die mit der Histogenese des Knorpels nichts zu tun haben und bei Knochen- und Elfenbeinzellen überhaupt nicht vorkommen.

\section{VI. Über die Analogie \\ in der Entwicklung der Knorpel-, Knochen- und Zahnbeingrundsubstanz und das Verhalten der Zellen zur Intercellularsubstanz.}

Naturgemäss werden die Bindegewebsarten, welche sich durch Festigkeit oder Widerstandsfähigkeit gegen Druck und Zug oder aber auch bei nur geringer Festigkeit durch einen hohen Grad von Elastizität auszeichnen, in eine besondere Klasse des Bindegewebes, in die der bindegewebigen Stützsubstanzen zusammengefasst. Indessen nicht nur ihrer Funktion, sondern auch der Struktur nach, haben die hierher gehörigen Gewebe des Knorpels, Knochens und Zahnbeins viel Gemeinsames. Wenn wir von den feinsten histologischen Strukturen absehen, so liegt die Übereinstimmung ihrer Bauart in dem Gefüge der Intercellularsubstanz. Diese Intercellularsubstanz oder Grundsubstanz der Autoren bildet ein Geflecht oder ein Balkenwerk von sich durchflechtenden oder überkreuzenden Bindegewebsfibrillen und Fibrillenbündeln. Die gegenseitige DurchHlechtung der Grundsubstanzfibrillen garantiert die Widerstandsfähigkeit gegen Druck und Zug: das Balkenwerk der Grundsubstanzfibrillen erhält durch die Kitt$\operatorname{substan} z$ oder homogene Interfibrillarsubstanz eine Versteifung, die durch die Kalksalzeinlagerung noch bedeutend erhöht werden kann. In Anbetracht der funktionellen Bedeutung der Grundsubstanz oder Intercellularsubstanz werden diese Gewebe auch Grundsubstanzgewebe genannt.

Aus meinen Arbeiten über die Histogenese der Zahnbeinund Knochengrundsubstanzen (Arch. f. mikr. Anat., Bd. 67 und Bd. 69) geht hervor, dass sie nach demselben Prinzip angelegt werden wie der Knorpel Das Gemeinsame ihres Aufbaues liegt darin, dass die erste Anlage fibrillär ist, dass sich in dem immer dichter werdenden fibrillären Geflecht die Bindegewebszellen zu 
den Grundsubstanzzellen differenzieren, dass erst nach der Entwicklung dieser Zellen auf das erste fibrilläre, acidophile das zweite homogene basophile Stadium folgt, dass das Wachstum der Grundsubstanzen in die Dirke der Hauptsache nach durch Apposition neuer Fibrillen erfolgt, wobei sich (Knochen, Knorpel) neue Bindegewebszellen in typische Grundsubstanzzellen umwandeln, was bei Elfenbeinzellen nicht nötig ist.

Bezüglich der Einzelheiten in der Histogenese der bindegewebigen Stützsubstanzen bedürfen noch manche Punkte der Aufklärung; doch über den grossen Plan ihres Aufbaues dürfte nach den von mir gemachten Untersuchungen kaum noch Ungewissheit bestehen. Ich möchte glauben, dass vor allem vergleichend anatomische Studien noch weiter Klarheit auf dem etwas schwierigen Gebiete der Histogenese der Stützsubstanzen bringen. Es wird sich immer melir zeigen, dass bei einseitiger Verfolgung von Anschauungen, wie sie der Odontoblasten- und Osteoblasten- und Chondroblastentheorie zugrunde liegen, unser Erkennen um nichts weiter kommt, sondern dass sich eine Theorie auf der anderen aufbauen muss.

Welcher Art die Funktion der definitiven Grundsubstanzzellen ist, darüber sagen meine Untersuchungen nichts aus. Nur soviel lasst sich mit Bestimmtheit sagen, dass sie nicht im Sinne der Autoren Grundsubstanz bilden können, d. h. sie secernieren nicht eine primäre homogene Grundsubstanz. Immerhin wäre es nun möglich, - ich musste es sogar als wahrscheinlich hinstellen dass die Knorpel-, Knochen- und Elfenbeinzellen den nicht fibrillaren, sondern homogenen Teil der Grundsubstanz, die Kittsubstanz bilden, danach wären sie also doch als Grundsubstanzbildner im beschränkten Sinne, wenn man so will, anzusehen.

Die hier wiedergegebenen Figuren meiner Arbeit „über die Analogie in der Entwicklung der Iinochen- und Zahnbeingrundsubstanz der Saugetiere nebst kritischen Bemerkungen über die Osteoblasten- und Odontoblastentheorie "(1906) zeigen die fibrillären Anlagen der Grundsubstanzen von Knochen und Zahnbein in ganz ähnlicher Weise, wie ich es jetzt beim Knorpel beschrieben habe.

Bezüglich des Verhaltens der Grundsubstanzzellen zur fibrillären Intercellularsubstanz weisen meine Untersuchungen mit Bestimmtheit darauf hin, dass die Grundsubstanzfibrillen ebensogut wie die Zellen als selbständige Gebilde angesehen werden 
müssen, die eigenen Stoffwechsel haben und aus eigener Kraft wachsen und sich vermehren. Nicht nur den Zellen, sondern auch den Intercellularsubstanzen der Stützgewebe müssen wir ein eigenes, nicht etwa von den Zellen erborgtes Leben zuschreiben. Die Intensitat des Lebensprozesses muss sich bei den Zellen wie

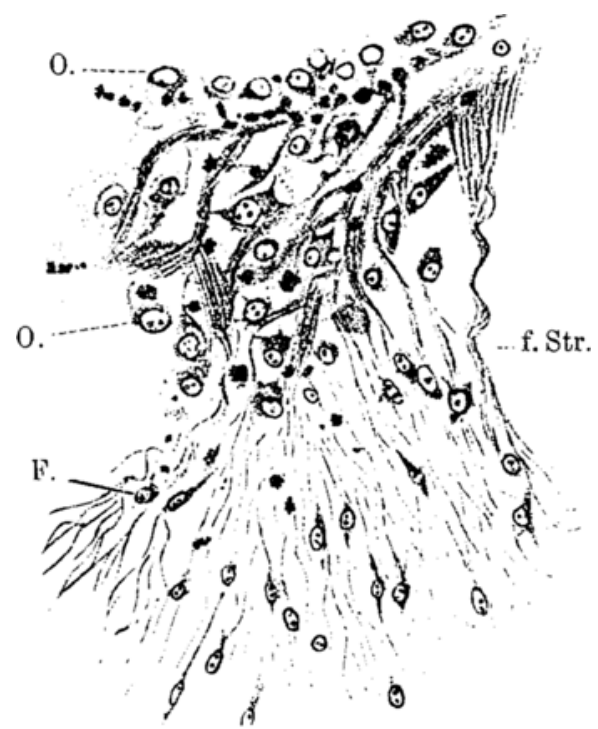

Fig. o.

Knochenanlage aus $v$. Korf $f$ (ds. Arch., Bd. 69, 1906, Taf. 19, Fig. 2).

0. = Osteoblasten; f. Str. = fibrillïre Stränge; F. = Fibroplasten.

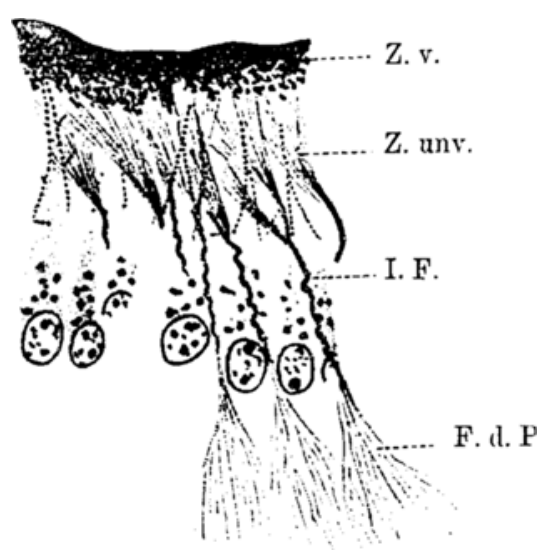

Fig. 6.

Zahnbeingrundsubstanzanlage aus v. Korff (ds. Arch., Bd. 69, 1906,

Taf. 19, Fig. 5).

F. d. P. = Fibrillen der Pulpa;

I. F. = Intercellulïre Fasern;

Z. unv. = Zahnbein unverkalkt; Z. v. = Zahnbein verkalkt.

bei den Intercellularsubstanzen nach den verschiedenen Stadien ihrer Entwicklung ändern. So besitzen zweifellos die heranwachsenden Knochen-, Knorpel- und Elfenbeinzellen einen hohen Grad der Lebenskraft, ebenso die jungen sich stark vermehrenden Bindegewebsfibrillen der ersten Anlage der Intercellularsubstanz, dagegen nimmt im definitiven Zustande dieser Gewebe die Intensität des Lebens in den sehr unscheinbar werdenden Zellen sichtbar ab, auch den Grundsubstanzfibrillen der verkalkten Intercellularsubstanzen können wir jetzt keinen hohen Grad der lebendigen Kraft mehr beimessen; Zellen wie Fibrillen verlieren in der definitiven (verkalkten) Grundsubstanz das Vermögen der Regeneration durch Teilung, was die Transplantationen dieser Gewebe beweisen (Marchand 1901). 
Gegenüber der von R. Virchow vertretenen Anschauung, dass der Stoffwechsel der Intercellularsubstanz hauptsächlich von den Grundsubstanzzellen abhängig sei und jede Zelle mit ihrem Stoffwechsel ein Territorium der Grundsubstanz beherrsche, weist M. Heidenhain in "Plasma und Zelle" (I. Abt., 1907, S. 32) auf folgendes hin: „Das Leben selbst ist seinem Begriffe nach überall etwas Aktives und bedeutet, dass der lebendige Teil a uf Grund allgemeiner Vorbedingungen, welche aus der Umgebung stammen, seine Struktur und seine Funktion selbsttatig erhalt (Automatie des Lebens). Ein passives, eingeblasenes oder eingehauchtes Leben gibt es nicht, denn die speziellen Bedingungen des Lebensprozesses liegen nicht in der Umgebung, sondern in den Dingen selbst. Lebt also etwas, so lebt es schlechthin wie die Zelle selbst. Da nun aber Leben immer nur direkt von Leben stammen kann, so kann auch die Intercellularsubstanz durchaus nicht etwa primär in einen zwischen den Zellen befindlichen Raum hinein abgelagert oder abgeschieden und hinterdrein erst organisiert und mit Leben durchdrungen worden sein, sondern die lebendige Intercellularsubstanz muss in direkter Weise von dem lebendigen Leibe der Zellen sich ableiten. Sie kann als lebende Masse nur Teil von lebenden Teilen sein, wobei sie freilich aus dem Leibe der Zellen herausgetreten, durch weiteres Wachstum dem Volumen nach vermebrt und durch besondere Differenzierung der Struktur nach umgestaltet wird."

\section{VII. Über die Ergebnisse}

der Untersuchungen F. Marchands über den Knorpelund Knochenneubildungsprozess bei der Heilung der Knorpel- und Knochenwunden.

Genaue Angaben über den Knorpelentwicklungsprozess bei der Heilung von Knorpelwunden verdanken wir F. Marchand (1901); ich gebe hier die Fig. 75b aus F. Marchands Werk „Der Prozess der Wundheilung mit Einschluss der Transplantation“ (Deutsche Chirurgie) wieder, an welcher das erste fibrilläre und das $z$ weite homogenaussehende Stadium der Knorpelgenese zu sehen ist. las regenerierende Knorpelgewebe geht hervor, wie wir gleich nach F. M a r chands exakten Beschreibungen sehen werden, aus dem Bindegewebe des Perichondriums, nicht von den Knorpelrändern 
der klaffenden Knorpelwunde. Der Wundspalt ist rollständig von Bindegewebe ausgefüllt, das unmittelbar mit dem des Perichondriums zusammenhängt, nicht aber mit dem benachbarten linorpelgewebe, von dem es sich sehr deutlich unterscheidet. Im Bindegewebe der klaffenden linorpelwunde sind zahlreiche Faserzüge (Fibrillenbündel) zur Entwicklung gekommen, deren Anordnung verschieden ist, viele „spannen sich quer oder schrïg durch die

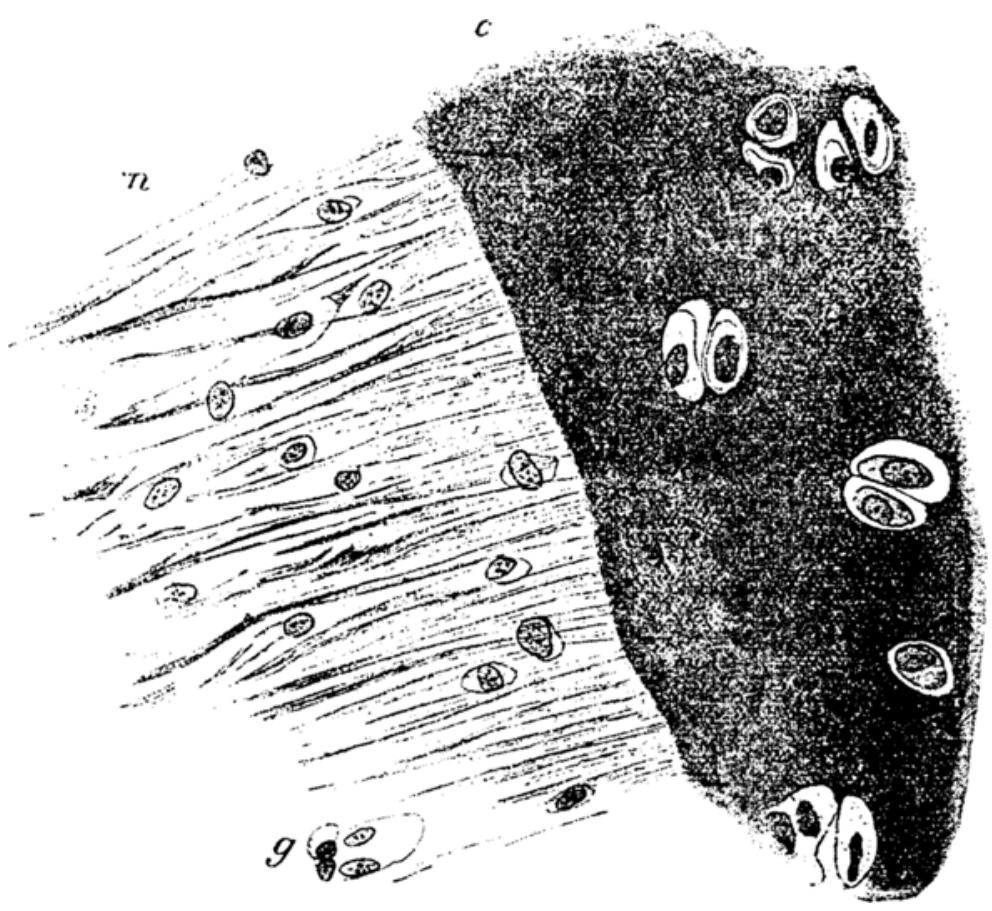

Fig. 7 aus F. Na r chand (1901).

$n=$ fibröses Narbengewebe einer 31 Tage alten Schnittwunde sines Rippenknorpels vom Hunde, das sich bei $\mathrm{c}$ in den unteren (älteren) bereits hyalinknorpligen Teil der Narbe umwandelt.

Wunde, von einer Seite zur anderen". „Von besonderem Interesse ist das Verhalten dieser Fasern zur Knorpelsubstanz; sie bilden meist Büschel, die pinselförmig in vollkommen gerade gestreckte Fasern auslaufen und mit ihren Enden ohne Grenze in die homogene Grundsubstanz des Knorpels übergehen. In den zwischen den einzelnen Büscheln frei bleibenden Lücken liegen ziemlich zerstreute rundliche Zellen; öfters ziehen die Fasern über die 
Zellen binweg, wodurch die Lücken verdeckt werden." "Obwohl die Fasern an dem meist ganz scharfen Rande des Wundspaltes mit der Grundsubstanz des Knorpels zusammenhüngen, gelingt es doch kaum an einer Stelle, sie in diese hinein zu verfolgen. Bei der oben angegebenen Färbung (Hämatoxylin-Pikrofuchsin) sind die Fasern ebenso wie die Knorpelsubstanz intensiv rot gefurbt, letztere nur in der Nähe der Knorpelzellen etwas blïulich. Man siebt nun häufig zwischen den roten Fasern in der Nähe des Knorpels eine ganz schwach bläulich gefarbte homogene Substanz auftreten, welche die Stelle, wo Zellen eingelagert sind, frei lässt. An etwas weiter vorgeschrittenen Stellen sind die Fasern in der Nachbarschaft des Knorpelrandes nicht mehr als solche erkennbar; mit der erwähnten Zwischensubstanz zu einer homogenen Masse verschmolzen, in welcher kleine rundliche helle Hohlrüume mit den darin eingelagerten Zellen sichtbar sind. Diese homogene Substanz hat bereits vollstandig die Beschaffenheit und Färbung. wie die Grundsubstanz des benachbarten Knorpels. In derselben Weise verhált sich die den Grund der Wundspalte einnehmende hyalin-knorpelige Masse, die sich nur durch einen geringen Unterschied in der Fărbung und etwas kleinere, dichter gedrinngte Zellen von dem alten Knorpel unterscheiden. Deutliche Proliferationserscheinungen sind am letzteren nicht vorhanden. Die spärlich abgestorbenen Knorpelzellen am Rande der Spalte sind verschwunden."

Über den Knochenbildungsprozess äussert sich F. March and (l. c., S. 280, 281) auf Grund von Präparaten der neugebildeten Knochensubstanz in den Markrüumen eines implantierten Schädelknochens: "Wir sehen zunächst aus den gewucherten jungen Spindelzellen Fibrillen hervorgehen, die immer mehr an Masse zunehmen und sich stellenweise zu einer anfangs noch deutlich faserigen, später mehr homogenen Masse in Gestalt schmaler oft schon frühzeitig netzförmig angeordneter Bälkchen verdichten. Inzwischen sind die Zellkörper von der fibrillären Substanz vollkommen abgelöst und bleiben als unregelmässige eckige, rundliche, langgestreckte Gebilde, teilweise in Lücken des Bälkchens, teilweise an seinen Rändern, sichtbar. Die ersteren würden schon als Knochenkörperchen zu bezeichnen sein, obwobl sie noch keine eigentliche Sternform mit feinen Ausläufern besitzen. Eine Unterscheidung dieser Zellen von den noch freiliegenden Osteoplasten 
ist nicht möglich." "Da, wo die junge Knochensubstanz sich an bereits vorhandene (z. B. abgestorbene) Knochenbälkchen anlagert, ist sie ebenfalls faserig, von derselben Beschaffenheit, wie an den freiliegenden Bälkchen. An der subduralen neugebildeten Osteophytschicht ist die junge Knochensubstanz ebenfalls, wemn auch nicht so deutlich, faserig."

Die Ergebnisse der Untersuchungen F. Marchands, welche mir leider erst jetzt bekannt geworden sind - ich hätte sie sonst früher in meinen Arbeiten erwähnt - stimmen durchaus mit dem, was ich in meinen früheren Arbeiten über die Bildung der Elfenbein- und Knochengrundsubstanz und in dieser über die des Knorpels hervorgehoben habe, überein, nämlich in der fibrillären Anlage der Grundsubstanzen, die erst später homogen erscheint (homogenisiert wird).

F. Marchand (l. c.) bemerkt schliesslich: „Der ganze Prozess der Knochenbildung aus Bindegewebe erinnert im hohen Grade an die Neubildung des hyalinen Knorpels aus gewuchertem Perichondrium; auch hier sehen wir die collagenen Fibrillen durch eine homogene Substanz vereinigt."

Dass die endochondrale Knochenbildung nach demselben Prinzip wie die periostale vor sich geht, lïsst sich leicht bei der Ossification der Geweihstangen verfolgen. Hierüber werde ich später berichten. Erwähnen will ich noch, dass nach Transplantationen Knorpelstücke ohne Perichondrium (vergl. F. Marchand, l. c., S. $451 \mathrm{ff}$.) nicht einheilen oder weiterwachsen, sondern resorbiert werden, da offenbar die typischen (definitiven) Knorpelzellen keine Proliferationsfähigkeit haben; die Transplantation der mit Perichondrium versehenen Knorpelstücke führt dagegen zur Neubildung. von Knorpelgewebe vom Bindegewebe des Perichondriums, nicht aber von den Knorpelzellen aus; auch hier geht das alte Knorpelgewebe zugrunde, wird resorbiert. Prinzipiell dieselben Resultate haben Transplantationen frischer (lebender) Knochenstücke. Nur mit Periost, das in der Zusammensetzung durchaus dem Perichondrium gleicht, überzogene Knochenstücke, auch ganze Knochen, bilden neuen Knochen von Periost aus, der alte Knochen dagegen wird vollkommen resorbiert, was natürlich nicht nötig wäre, wenn die alten Knochenzellen oder auch die sogenannten Osteoblasten Knochensubstanz bilden könnten. Hiermit ist aber eine natürliche Erklärung gefunden für die vergeblichen 
Versuche, Knochen- oder Knorpelwunden durch Transplantationen von frischen Knorpel- und Knochenstücken schnell zur Heilung zu bringen, etwa so, wie es bei Hautwunden oder Hautdefekten durch Transplantation von Hautlappen möglich ist, wobei nach baldiger Abstossung der obertlachlichen Lagen der Epidermiszellen die Epithelzellen des Stratum germinativum durch Zellwucherung neue Zellschichten produzieren und auch im Bindegewebe der transplantierten Cutis keimende (in mitotischer Teilung begriffene) Bindegewebszellen und gut erhaltene collagene und elastische Bindegewebsfasern konstatiert wurden (F. Marchand, 1. c., S. 420 ff.).

\section{Literaturverzeichnis.}

Bigelow, W. S.: Notiz über den Teilungsvorgang bei Knorpelzellen sowie über den Bau des Hyalinknorpels. Arch. f. mikr. Anat., Bd. 16, 1879. Claparède, E.: Anatomie der Neretina Huviatilis. M ìlle r's Arch., 1857. Hammar, J. A u g.: Über den feineren Bau der Gelenke. I. Die Gelenkmembran. II. Der Gelenkknorpel. Arch. f. mikr. Anat., Bd. 43, 1894.

$\mathrm{H}$ ansen, F. C. C.: Untersuchungen über die Gruppe der Bindesubstanzen. I. Der Hyalinknorpel. Anat. Hefte, H. 83, Bd. 27, 1905.

Hasse: Über den Bau und die Entwicklung des hyalinen Knorpels bei den Elasmobranchiern. Zool. Anz., 1879.

Derselbe: Das natürliche System der Elasmobranchier. Jena 1879-1882. Heidenhain, R.: Studien aus dem physiologischen Institute zu Breslau. H. 1, 1861; H. 2, 1863.

Heidenhain, M.: Plasma und Zelle. I. Abt. Allgemeine Anatomie der lebendigen Masse. 1. Lieferung. Jena 1907.

Hertwig, O.: Über die Entwicklung und Bau des elastischen Gewebes im Netzknorpel. Arch. f. mikr. Anat., Bd. 9, 1873.

Koelliker, A.: Handbuch der Gewebelehre des Menschen. 6. Auflage, Bd. I, Leipzig 1889.

v. Korff, K.: Zur Histologie und Histogenese des Bindegewebes besonders der Knochen und Dentingrundsubstanz. Erg. d. Anat. u. Entwicklungsgeschichte von Merkel u. Bonnet, 17. Bd., 1907.

Derselbe: Die Entwicklung der Zahnbeingrundsubstanz der Säugetiere. Arch. f. mikr. Anat., Bd. 67, 1905.

Derselbe: Die Analogie in der Entwicking der Knochen- und Zahnbeingrundsubstanz der Säugetiere nebst kritischen Bemerkungen über die Osteoblasten- und Odontoblastentheorie. Arch.f. mikr. Anat., Bd. 69, 1906

Derselbe: Entgegnung auf die v. E bnersche Abhandlung „Über scheinbare und wirkliche Radiärfasern des Zahnbeins". Anat. Anz., Bd. 34, 1909.

Derselbe: Zur Histogenese der bindegewebigen Stützsubstanzen niederer Wirbeltiere. Verhandlg. d. anat. Gesellsch., 1910. 
Kruckenberg, C. Fr. W.: Die chemischen Bestandteile des Knorpels. Zeitschr. f. Biol., Bd. 20, 1884.

Harchand, F.: Der Prozess der Wundheilung mit Einschluss der Transplantation. Deutsche Chirurgie, 16. Bd., 1901.

Mörner, C. Th.: Histochemische Beobachtungen über die hyaline Grundsubstanz des Trachealknorpels. Zeitschr. f. phys. Chemie, Bd. 12, 1888.

Derselbe: Chemische Studien über den Trachealknorpel. Skandin. Arch. $f$. Phys., Bd. 1, 1889.

Renaut: Traité d'histologie pratique. Tome 1. Paris 1893.

Schaffer, Jos ef: Über den feineren Bau und die Entwicklung des Knorpelgewebes und über verwandte Formen der Stützsubstanzen. I. Teil. Zeitschr. f. wiss. Zool, Bd. 70, 1901.

Schleicher, W.: Die Knorpelzellteilung. Arch. f. mikr. Anat., Bd. 16, 1879.

Schmiedeberg, O.: Über die chemische Zusammensetzung des Knorpels. Arch. f. experiment. Path. u. Pharmak., Bd. 28, 1891.

Schwalbe: Knorpelregeneration und Knorpelwachstum. Sitzungrber. d. Jenaischen Ges. f. Med. u. Naturw., Jahrg. 1878.

Sieveking, H.: Beiträge zur Kenntnis des Wachstums nnd der Regeneration des Knorpels nach Beobachtungen am Kaninchen- und Mïuseolrr. Morph. Arbeiten, herausgeg. von G. Schwalbe, 1. Bd., 1892.

Spuler, Arnold: Ûber Bau und Entstehung des elastischen Netzlnorpels. Sitzungsber. d. physik.-med. Ges. zu Erlangen, 27. Heft, 1895.

Derselbe: Beitrïge zur Histologie und Histogenese der Binde- und Stützsubstanzen. Anat. Hefte, Bd. 7, 1896.

Strasser: Zur Entwicklung des Extremitätenknorpels bei Salamandern und Tritonen. Morph. Jahrb., Bd. V.

van der Stricht, Omer: Recherches sur le cartilage hyalin. Arch. de Biologie (de Gand), Tome 7, 1887.

Studnička, F. K.: Bemerkungen über die Histologie und Histogenese des Knorpels der Cyclostomen. Arch. f. mikr. Anat., Bd. 48, 1897.

Derselbe: Weitere Bemerkungen über das Knorpelgewebe der Cyclostomen und seine Histogenese. Arch. f. mikr. Anat., Bd. כ̌l, 1898.

Derselbe : Histologische und histogenetische Untersuchungen über das Knolpel-, Vorknorpel- und Chordagewebe. Anat. Hefte, 21. Bd. (66. u. 67. Heft), 1903.

Tillmanns, H.: Über die fibrilläre Struktur des Hyalinknorpels. Arch. f. mikr. Anat. u. Phys., Anat. Abt., 1877. 


\section{Erklärung der Abbildungen auf Tafel XI.}

$\mathrm{a}=$ Perichondrium $; \mathrm{b}=$ Vorknorpel $; \mathrm{c}=$ Knorpel $; \mathrm{F}=$ Fibroblasten $;$ $\mathrm{VK}=$ Vorknorpelzellen $; \mathrm{K}=$ Knorpelzellen: $\mathrm{FL}=$ fibrilläre Lamellen.

Fig. 1. Äussere Schicht des Perichondriums des Hyalinknorpels der Salamanderlarve (Kiemenknorpel). Flachschnitt. Grosse, meist langgestreckte Fibroblasten. Zelleib blass, hat zahlreiche Körner und Körnerketten und Plasmafibrillen differenziert. Zwischen den Zellen präcollagene Bindegewebsfibrillen. Z en ker sche Flüssigkeit. Eisenalaunhämatoxylin. Obj. Z e is s D, Ocul. 4. L = Leucocyt.

Fig. 2. Hyalinknorpel des Schädels der Salamanderlarve.

a) Zone des Perichondriums. Fibroblasten nach der Fibrillenbildung. Kerne plasmaarm oder ${ }_{n}$ nackit. Die Fibrillen sich geflechtartig durchsetzend sind zur prochondralen Substanz geworden.

b) Zone des Vorknorpels. Zunahme der Intercellularsubstanz. In den Lücken zwischen den sich durchflechtenden Bindegewebstibrillenzügen der prochondralen Substanz differenzieren sich die Bindegewebszellen zu Vorknorpelzellen. Übergang der Fibrillen des Perichondriums in die des Vorknorpeis.

c) Zone des Knorpels mit Knorpelhöhlen, Knorpelzellen und einer mehr homogen aussehenden Intercellularsubstanz. Übergang der prochondralen Substanz in den Knorpel. Zenkersche Flüssigkeit. Mallory färbung. Obj. $Z$ e is s D, Ocul. 4.

Fig. 3. Schrägschnitt des Kiemenknorpels der Salamanderlarve. Zen kersche Flüssigkeit. Mallor y färbung. Das Perichondrium auf der einen Seite flach geschnitten, zeigt eine Zusammensetzung aus fibrillären, der Oberfläche mehr oder weniger parallel laufenden Lamellen. Zwischen den Lamellen die plasmaarmen Bindegewebszellen nach der Fibrillenbildung. Auf der anderen Seite sind die fibrillären Lamellen quer geschnitten. Am Knorpelrande Übergang der Bindegewebsfibrillen des Perichondriums in die Knorpelgrundsubstanz. Vorknorpelzellen mit grossem Kern, der immer kleiner wird und schmalem Plasmastreif, der grösser wird. Obj. Zeiss D, Ocul. 4.

Fig. 4. Flachschnitt durch den Knorpelrand der Scapula einer Salamanderlarve. Zenkersche Flüssigkeit. Eisenalaunhämatoxylin. Übereinander liegende fibrilläre Lamellen des Perichondriums, die in die Grundsubstanz des Knorpels verfolgbar sind, wo ihre fibrilläre Struktur mehr und mehr verschwindet. Die zwischen den Lamellen liegenden Vorknorpelzellen in mitotischer Teilung. (Stadium des lockeren Knäuels und des Muttersterns.) Obj. Z eiss D, Ocul. 4.

Fig. 5. Schrägschnitt durch die Randpartie des Hyalinknorpels der Selachier (Acanthias vulgaris ca. $15 \mathrm{~cm}$ lang), Schädeldach. Z enkersche Flüssigkeit. Mall or y färbung. $\mathrm{FL}=$ Lamellenbildung der Fibrillen des Perichondriums. Hauptlamellen und sich abzweigende oder durchsetzende Nebenlamellen. Die Fibrillen der Lamellen sind beim 
Über die Histogenese und Struktur der Knorpelgrundsubstanz. 299

Übergang in die Knorpelgrundsubstanz weniger färbbar. Die plasmaarmen Bindegewebszellen zwischen den Lamellen regenerieren ihren Zelleib, der allmählich an Grösse zunimmt; sie werden zu Knorpelzellen. Obj. Zeiss D, Ocul. 4.

Fig. 6. Hyalinknorpel. Selachier. Acanthias vulgaris ca. $10 \mathrm{~cm}$ lang. Zenkersche Flüssigkeit. Hämalaun. Benzolichtbordeaux. In den oberflächlichen Schichten zwischen den Bindegewebszellen das erste Stadium der Chondrogenese als ein Geflecht von acidophilen Bindegewebsfibrillen, fibrilläre prochondrale Substanz. Am Knorpelrande zwischen den Vorknorpel- bezw. Knorpelzellen das zweite Stadium der Chondrogenese. Hyalinwerden der Intercellularsubstanz durch die basophile Kittsubstanz. Maskierung der Bindegewebsfibrillen. Obj. Zeis s D, Ocul. 4 .

Fig. 7. Gelenkknorpel; neugeborener Hund. Fle mming sche Flüssigkeit. II a ll o ry fürbung. Kontinuierlicher Übergang der inneren Schichten des faserigen Perichondriums in die Grundsubstanz des Knorpels. Aussen lamelläre Anordnung der Bindegewebsfibrillen des Perichondriums (Hauptlamellen der Oberfläche, parallel dazwischen Nebenlamellen), Verbreiterung der lamellenartigen Intercellularsubstanz am Knorpelrande. Lamellüre Anordnung der Fibrillen verschwindet beim Übergang der Fibrillen in die Knorpelgrundsubstanz. Objekt Zeis s D, Ocul. 4. 


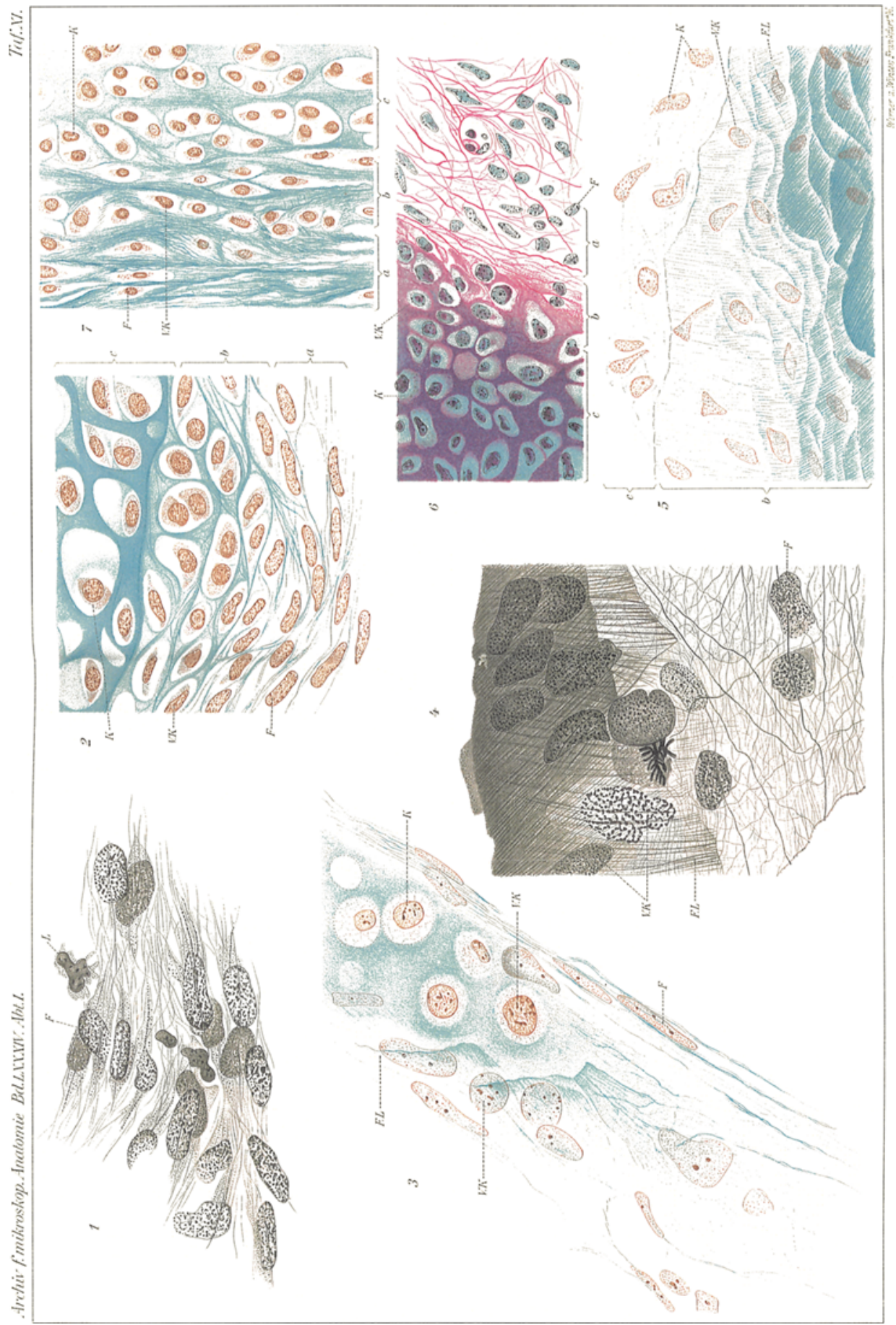

